\title{
A Review of Unsupervised Spectral Target Analysis for Hyperspectral Imagery
}

\author{
Chein-I Chang, ${ }^{1,2}$ Xiaoli Jiao, ${ }^{1}$ Chao-Cheng Wu, ${ }^{1}$ Yingzi Du, ${ }^{3}$ and Mann-Li Chang ${ }^{4}$ \\ ${ }^{1}$ Remote Sensing Signal and Image Processing Laboratory, Department of Computer Science and Electrical Engineering, \\ University of Maryland, Baltimore, MD 21250, USA \\ ${ }^{2}$ Department of Electrical Engineering, National Chung Hsing University, Taichung, Taiwan \\ ${ }^{3}$ Department of Electrical and Computer Engineering, Purdue School of Engineering and Technology, \\ Indiana University-Purdue University Indianapolis, Indianapolis, IN 46202, USA \\ ${ }^{4}$ Management and Information Department, Kang Ning Nursing and Management Junior College, Taipei, Taiwan
}

Correspondence should be addressed to Chein-I Chang, cchang@umbc.edu

Received 27 September 2009; Revised 31 December 2009; Accepted 19 February 2010

Academic Editor: Jin-Hua She

Copyright ( $) 2010$ Chein-I Chang et al. This is an open access article distributed under the Creative Commons Attribution License, which permits unrestricted use, distribution, and reproduction in any medium, provided the original work is properly cited.

\begin{abstract}
One of great challenges in unsupervised hyperspectral target analysis is how to obtain desired knowledge in an unsupervised means directly from the data for image analysis. This paper provides a review of unsupervised target analysis by first addressing two fundamental issues, "what are material substances of interest, referred to as targets?" and "how can these targets be extracted from the data?" and then further developing least squares (LS)-based unsupervised algorithms for finding spectral targets for analysis. In order to validate and substantiate the proposed unsupervised hyperspectral target analysis, three applications in endmember extraction, target detection and linear spectral unmixing are considered where custom-designed synthetic images and real image scenes are used to conduct experiments.
\end{abstract}

\section{Introduction}

Hyperspectral imaging has become an emerging technique in remote sensing analysis. With high spectral resolution many material substances which are not known a priori or cannot be visualized by inspection can now be revealed by hyperspectral imaging sensors for data exploitation. Consequently, two main issues are investigated in this paper. One is what are material substances of interest? Once targets of interest are defined, the next is how to find these targets directly from the data in an unsupervised manner without prior knowledge. In order to address the first issue, we first explore a new concept of so-called "spectral" targets which is developed to differentiate targets commonly addressed in traditional image processing. With no spectral bands used in traditional image processing the targets of interest are generally identified by their spatial properties such as size, shape, and texture. In this case, targets to be recognized based on their spatial properties can be considered as "spatial" targets and the techniques developed to recognize such spatial targets are referred to as spatial domain-based image processing techniques. On the other hand, due to use of spectral bands specified by a range of wavelengths a multispectral or hyperspectral image pixel is actually a column vector, of which a pixel of spectral band is produced by a particular wavelength. As a consequence, a single image pixel vector of a hyperspectral image already contains abundant spectral information provided by hundreds of contiguous spectral bands that can be used for data exploitation. Such spectral information within a single image pixel vector is referred to as intrapixel spectral information. A target analyzed based on its spectral properties characterized by the intrapixel spectral information on a single image pixel vector basis is called "spectral target" as opposed to "spatial target" analyzed by interpixel spatial information provided by spatial correlation among sample pixels. More specifically, three major types of spectral targets are of particular interest in this paper. One is endmembers whose spectral signatures are idealistically pure [1]. Endmembers do not usually appear in multispectral images due to low spatial and spectral resolution but have 
become increasingly important in hyperspectral imaging because an endmember can be used to identify a spectral class. Another is subpixel targets which do not fully occupy a pixel but rather are completely embedded in a single image pixel vector [2]. This type of targets cannot be visualized spatially and can be only recognized based on their spectral properties. Subpixel targets occur when their spatial extent is smaller than pixel resolution. A third type of targets is mixed targets whose spectral signatures are linearly or nonlinearly mixed by a number of target spectral signatures with appropriate portions of fractions present in a single image pixel vector [2]. The occurrence of a mixed target is a result of low spatial and spectral resolution and it may partially occupy more than one pixel vector. Apparently, none of these three types of spectral targets can be effectively analyzed by spatial domain-based techniques.

With a spectral target defined as above what we are particularly interested in this paper from an aspect of statistical signal processing are two types of spectral targets, one characterized by 2 nd order sample intrapixel Spectral Information Statistics (SIS) and the other by sample intrapixel SIS of order higher than 2 , referred to as high-order SIS. It should be noted that the term of sample intrapixel SIS is defined as correlation of intrapixel SIS among samples. In the context of sample spectral information statistics we assume that background (BKG) pixels are those spectral targets characterized by 2 nd sample intrapixel SIS while the target pixels of interest are those high-order sample intrapixel SIS. In hyperspectral image analysis this seems a reasonable assumption since the spectral targets of interest in hyperspectral data exploitation are those which either (1) occur with low probability or (2) have small populations when they are present. In other words, these types of spectral targets are usually relatively small, appear in small population and also occur with low probabilities, for example, special spices in agriculture and ecology, toxic wastes in environmental monitoring, rare minerals in geology, drug/smuggler trafficking in law enforcement, combat vehicles in the battlefield, landmines in war zones, chemical/biological agents in bioterrorism, weapon concealment and mass graves. These spectral targets are generally considered as insignificant objects because of their very limited spatial information but they are actually critical and crucial for defense and intelligence analysis since they are insignificant compared to targets with large sample pools and generally hard to be identified by visual inspection and, from a statistical point of view, the spectral information statistics of such special targets cannot be captured by 2 nd order sample intrapixel SIS but rather by high-order sample intrapixel SIS.

Once image pixel vectors are categorized into BKG and target classes according to sample intrapixel SIS, a followup task is how to find them in which case two issues need to be addressed. One is how many of them. The other is how to extract them. The first issue can be resolved by a new concept, virtual dimensionality (VD) recently developed [2, Chapter 17], [3]. The idea of VD is based on the assumption that if a signal source is presenting in the data, it will contribute energy to 1st order statistics. In doing so, both the eigenvalues of sample correlation matrix, $\left\{\hat{\lambda}_{l}\right\}$ and the eigenvalues of covariance matrix, $\left\{\lambda_{l}\right\}$ are calculated. If $\hat{\lambda}_{l}-\lambda_{l}$ is greater than zero resulting from the $l$ th component sample mean, it implies that there is a signal source; otherwise, no signal is present. To materialize this idea, a binary composite hypothesis testing problem is formulated in a way that the null and alternative hypotheses, $H_{0}$ and $H_{1}$ represent two scenarios, $H_{0}: \hat{\lambda}_{l}-\lambda_{l}=0$ and $H_{1}: \hat{\lambda}_{l}-\lambda_{l}>0$, respectively. The Neyman Pearson detection theory [4] is then applied to find how many times the test fails running over all spectral bands for a given false alarm probability, $P_{F}$. It is the number of test failures that indicates the number of signal sources assumed to be in the data. The beauty of VD lies on the fact that its value is completely determined by the $P_{F}$. By varying the value of $P_{F}$, the number of spectrally distinct signatures estimated by the VD varies. For example, if $P_{F}$ is set low, fewer tests will fail and thus fewer targets are assumed to be in the data and vice versa. To address the second issue an unsupervised spectral target finding algorithm (USTFA) is developed which is based on three least squares (LS)-based algorithms, automatic target generation process (ATGP) [5], unsupervised nonnegativity constrained least squares (UNCLS) method [6] and unsupervised fully constrained least squares method (UFCLS) [7]. In order for these unsupervised methods to extract and distinguish spectral targets of 2nd order sample intrapixel SIS from high-order sample intrapixel SIS, two data sets, original data and its sphered data are used. It assumes that the BKG in a hyperspectral image is most likely characterized by 2 nd order sample intrapixel SIS while hyperspectral targets will be more likely to be captured by high-order sample intrapixel SIS as outliners due to their small spatial presence. In this case, high-order spectral targets are referred to as desired targets to be used for image analysis, while 2nd order spectral targets are considered as undesired targets for which we would like to annihilate or suppress prior to data processing so as to improve image analysis.

In order to validate the utility of VD and the proposed unsupervised spectral target analysis for hyperspectral imagery, custom-designed synthetic image experiments with complete ground truth are conducted to show that the VD indeed provides a reasonable estimate of the true dimensionality for qualitative and quantitative analysis for three applications, endmember extraction, unsupervised spectral target detection and unsupervised LSU-based target classification. These same experiments are further substantiated by real image data.

As summarized, several contributions are made in this paper. First and the foremost is to introduce the concept of sample intrapixel SIS to define BKG and target pixels characterized by 2 nd order sample intrapixel SIS $s$ and high-order sample intrapixel SIS, respectively. A second contribution is to use the VD to determine the number of BKG and target pixels, each of which represents a particular spectral class, either a BKG or a target class. A third contribution is to come up an idea of using two sets of original data and its sphered data from which BKG and target pixels can be extracted. A fourth contribution is to design an USTFA to extract BKG and target pixels. Finally, a fifth contribution is to custom 
design synthetic image experiments to validate the proof of concept for the unsupervised target analysis developed in this paper. This is then followed by real image experiments to substantiate its utility in real applications.

\section{Unsupervised Least Squares-Based Algorithms}

In this section, we present three least squares (LS)-based algorithms for finding spectral targets of interest using a posteriori knowledge which can be obtained directly from the data.

To accomplish this task, a designed least squares-based algorithm is first applied to the original data to extract data sample vectors characterized by 2 nd order SIS. Then, the same algorithm is further applied to the data which is sphered by removing the data sample mean and covariances while making data variances ones so that all 2 nd order SIScharacterized data samples will be on the sphere and all other data sample vectors that are characterized by high-order SIS are either inside (sub-Gaussian samples) or outside the sphere (super-Gaussian samples). As a consequence of such a sphering process, the resulting data has the 1 st and 2nd order SIS removed from the original data because of zero mean and constant variance one so that samples characterized by SIS of orders higher than 2 can be extracted from inside or outside the sphere. Interestingly, despite that the idea of using the same algorithm in two passes, one pass for the original data and another pass for the sphered data to extract two types of targets of interest, 2nd order targets and high-order targets for data analysis seem simple, it is by no means a trivial matter because its novelty has never been explored in the open literature. In what follows, we design and develop three LS-based algorithms for this purpose.

The first algorithm of interest was previously proposed by Ren and Chang in [5], called Automatic Target Generation Process (ATGP) which can be considered as an unsupervised version of the orthogonal subspace projection (OSP) algorithm in [8]. Its relationships with LS-based linear spectral unmixing (LSU) were also explored in $[9,10]$. With this interpretation, the ATGP can be also viewed as an unsupervised version of an unconstrained LS LSU method. The 2nd and 3rd LS-based algorithms are an unsupervised version of a partially abundance-constrained least squares LSU, referred to as Unsupervised Non-negativity Constrained Least Squares (UNCLS) [6] and an unsupervised version of fully abundance least squares LSU, referred to as unsupervised fully constrained least squares (UFCLS) [7].

Assume that $\mathbf{m}_{1}, \mathbf{m}_{2}, \ldots, \mathbf{m}_{p}$ are spectral signatures used to unmix the data sample vectors. Let $L$ be the number of spectral bands and $\mathbf{r}$ be an $L$-dimensional data sample vector which can be modeled as a linear combination of $\mathbf{m}_{1}, \mathbf{m}_{2}, \ldots, \mathbf{m}_{p}$ with appropriate abundance fractions specified by $\alpha_{1}, \alpha_{2}, \ldots, \alpha_{p}$. More precisely, $\mathbf{r}$ is an $L \times 1$ column vector and $\mathbf{M}$ is an $L \times p$ target spectral signature matrix, denoted by $\left\lfloor\mathbf{m}_{1} \mathbf{m}_{2} \cdots \mathbf{m}_{p}\right\rfloor$, where $\mathbf{m}_{j}$ is an $L \times 1$ column vector represented by the spectral signature of the $j$ th target resident in the pixel vector $\mathbf{r}$. Let $\boldsymbol{\alpha}=\left(\alpha_{1}, \alpha_{2}, \ldots, \alpha_{p}\right)^{T}$ be a $p \times 1$ abundance column vector associated with $\mathbf{r}$ where $\alpha_{j}$ denotes the fraction of the $j$ th target signature $\mathbf{m}_{j}$ present in the pixel vector $\mathbf{r}$. A classical approach to solving a mixed pixel classification problem is linear unmixing which assumes that the spectral signature of the pixel vector $\mathbf{r}$ is linearly mixed by $\mathbf{m}_{1}, \mathbf{m}_{2}, \ldots, \mathbf{m}_{p}$ as follows:

$$
\mathbf{r}=\mathbf{M} \boldsymbol{\alpha}+\mathbf{n},
$$

where $\mathbf{n}$ is noise or can be interpreted as a measurement or model error.

Equation (1) represents a standard signal detection model where $\mathbf{M} \boldsymbol{\alpha}$ is a desired signal vector needed to be detected and $\mathbf{n}$ is a corrupted noise. Since we are interested in detecting one target at a time, we can divide the set of the $p$ target signatures, $\mathbf{m}_{1}, \mathbf{m}_{2}, \ldots, \mathbf{m}_{p}$ into a desired target, say $\mathbf{m}_{p}$ and a class of undesired target signatures, $\mathbf{m}_{1}, \mathbf{m}_{2}, \ldots, \mathbf{m}_{p-1}$. In this case, a logical approach is to eliminate the effects caused by the undesired targets $\mathbf{m}_{1}, \mathbf{m}_{2}, \ldots, \mathbf{m}_{p-1}$ that are considered as interferers to $\mathbf{m}_{p}$ before the detection of $\mathbf{m}_{p}$ takes place. With annihilation of the undesired target signatures the detectability of $\mathbf{m}_{p}$ can be therefore enhanced. In doing so, we first separate $\mathbf{m}_{p}$ from $\mathbf{m}_{1}, \mathbf{m}_{2}, \ldots, \mathbf{m}_{p}$ in $\mathbf{M}$ and rewrite (1) as

$$
\mathbf{r}=\mathbf{d} \alpha_{p}+\mathbf{U} \gamma+\mathbf{n},
$$

where $\mathbf{d}=\mathbf{m}_{p}$ is the desired spectral signature of $\mathbf{m}_{p}$ and $\mathbf{U}=$ $\left\lfloor\mathbf{m}_{1} \mathbf{m}_{2} \cdots \mathbf{m}_{p-1}\right\rfloor$ is the undesired target spectral signature matrix made up of $\mathbf{m}_{1}, \mathbf{m}_{2}, \ldots, \mathbf{m}_{p-1}$ which are the spectral signatures of the remaining $p-1$ undesired targets. Using (2) we can design an orthogonal subspace projector to annihilate $\mathbf{U}$ from the pixel vector $\mathbf{r}$ prior to detection of $\mathbf{t}_{p}$. One of such desired orthogonal subspace projectors was the orthogonal subspace projection (OSP) derived in [8] and given by

$$
P_{\mathbf{U}}^{\perp}=\mathbf{I}-\mathbf{U U}^{\#},
$$

where $\mathbf{U}^{\#}=\left(\mathbf{U}^{T} \mathbf{U}\right)^{-1} \mathbf{U}^{T}$ is the pseudo-inverse of $\mathbf{U}$. The notation $\frac{1}{\mathbf{U}}$ in $P_{\mathbf{U}}^{\perp}$ indicates that the projector $P_{\mathbf{U}}^{\perp}$ maps the observed pixel vector $\mathbf{r}$ into the orthogonal complement of $\langle\mathbf{U}\rangle$, denoted by $\langle\mathbf{U}\rangle^{\perp}$. By means of (3) a linear optimal signal detector for (2), denoted by $\delta^{\mathrm{OSP}}(\mathbf{r})$ was developed in [8] and given by

$$
\delta^{\mathrm{OSP}}(\mathbf{r})=\mathbf{d}^{T} P_{\mathrm{U}}^{\perp} \mathbf{r}
$$

2.1. Automatic Target Generation Process (ATGP). The ATGP can be considered as an unsupervised and unconstrained OSP technique which performs a succession of orthogonal subspace projections specified by (3) to find a set of sequential data sample vectors that represents targets of interest as follows.

\section{Automatic Target Generation Process (ATGP) Algorithm.}

(1) Initial condition:

Let $\varepsilon$ be a prescribed error threshold and $\mathbf{t}_{0}$ be a pixel with brightest intensity value, that is, largest gray level value. Set $k=0$. 
(2) Let $k \leftarrow k+1$ and apply $P_{\mathbf{t}_{0}}^{\perp}$ via (3) to all image pixels $\mathbf{r}$ in the image and find the $k$ th target $\mathbf{t}_{k}$ generated at the $k$ th stage which has the maximum orthogonal projection as follows:

$$
\mathbf{t}_{k}=\arg \left\{\max _{\mathbf{r}}\left\lfloor\left(P_{\left[\mathbf{U}_{k-1} \mathbf{t}_{k}\right]}^{\perp} \mathbf{r}\right)^{T}\left(P_{\left[\mathbf{U}_{k-1} \mathbf{t}_{k}\right]}^{\perp} \mathbf{r}\right)\right]\right\} .
$$

(3) If $m\left(\mathbf{t}_{k-1}, \mathbf{t}_{k}\right)>\varepsilon$ where $m(\cdot, \cdot)$ can be any target discrimination measure, for example, Spectral Angle Mapper (SAM) in [2], then go to step (2). Otherwise, the algorithm is terminated. At this point, all the generated target pixels $\mathbf{t}_{0}, \mathbf{t}_{1}, \ldots, \mathbf{t}_{k-1}$ are considered as the desired targets.

2.2. Unsupervised Non-Negativity Least Squares (UNCLS) Method. The UNCLS is an unsupervised version of the abundance Non-negativity Constrained Least squares (NCLS) where the NCLS is a partially abundance unconstrained OSP technique that imposes the abundance non-negativity constraint (ANC), $\boldsymbol{\alpha} \geq \mathbf{0}$, that is, $\alpha_{j} \geq 0$ for all on the linear mixing model specified by (1). It can be implemented in the following.

\section{UNCLS Algorithm.}

(1) Initial condition:

Select $\varepsilon$ to be a prescribed error threshold and let $\mathbf{t}_{0}=$ $\arg \left\{\max _{\mathbf{r}}\left[\mathbf{r}^{T} \mathbf{r}\right]\right\}$ where $\mathbf{r}$ is run over all image pixel vectors, and set $k=0$.

(2) Let $\operatorname{LSE}^{(0)}(\mathbf{r})=\left(\mathbf{r}-\hat{\alpha}_{0}^{(1)}(\mathbf{r}) \mathbf{t}_{0}\right)^{T}\left(\mathbf{r}-\hat{\alpha}_{0}^{(1)}(\mathbf{r}) \mathbf{t}_{0}\right)$ and check if $\max _{\mathbf{r}} \mathrm{LSE}^{(0)}(\mathbf{r})<\varepsilon$. If yes, the algorithm is terminated; otherwise continue.

(3) Let $k \leftarrow k+1$ and find $\mathbf{t}_{k}=\arg \left\{\max _{\mathbf{r}}\left[\operatorname{LSE}^{(k-1)}(\mathbf{r})\right]\right\}$.

(4) Apply the NCLS method with the signature matrix $\mathbf{M}^{(k)}=\left[\mathbf{t}_{0} \mathbf{t}_{1} \cdots \mathbf{t}_{k-1}\right]$ to estimate the abundance fraction of $\mathbf{t}_{0}, \mathbf{t}_{1}, \ldots, \mathbf{t}_{k-1}, \hat{\alpha}_{1}^{(k)}(\mathbf{r}), \hat{\alpha}_{2}^{(k)}(\mathbf{r}), \ldots, \hat{\alpha}_{k-1}^{(k)}(\mathbf{r})$.

(5) Find the $k$ th maximum least squares error defined by

$$
\begin{aligned}
& \max _{\mathbf{r}}\left\{\operatorname{LSE}^{(k)}(\mathbf{r})\right\} \\
& =\max _{\mathbf{r}}\left\{\left(\mathbf{r}-\sum_{j=1}^{k-1} \hat{\alpha}_{j}^{(k)} \mathbf{t}_{j}\right)^{T}\left(\mathbf{r}-\sum_{j=1}^{k-1} \hat{\alpha}_{j}^{(k)} \mathbf{t}_{j}\right)\right\} .
\end{aligned}
$$

(6) If $\max _{\mathbf{r}} \mathrm{LSE}^{(k-1)}(\mathbf{r})<\varepsilon$, the algorithm is terminated; otherwise go to step (3).

2.3. Unsupervised Fully Least Squares (UFCLS) Method. The UFCLS is an unsupervised abundance Fully Constrained Least Squares (FCLS) where the FCLS is a partially abundance unconstrained OSP technique that imposes both Abundance Sum-to-one Constraint (ASC), that is, $\sum_{j=1}^{p} \alpha_{j}=$ 1 and Abundance Non-negativity Constraint (ANC), $\boldsymbol{\alpha} \geq$ $\mathbf{0}$, that is, $\boldsymbol{\alpha}_{j} \geq 0$ on the linear mixing model (1). Its implementation is provided below.

\section{UFCLS Algorithm.}

(1) Initial condition:

Select $\varepsilon$ to be a prescribed error threshold and let $\mathbf{t}_{0}=$ $\arg \left\{\max _{\mathbf{r}}\left[\mathbf{r}^{T} \mathbf{r}\right]\right\}$ where $\mathbf{r}$ is run over all image pixel vectors, and let $k=0$.

(2) Let $\operatorname{LSE}^{(0)}(\mathbf{r})=\left(\mathbf{r}-\hat{\alpha}_{0}^{(1)}(\mathbf{r}) \mathbf{t}_{0}\right)^{T}\left(\mathbf{r}-\hat{\alpha}_{0}^{(1)}(\mathbf{r}) \mathbf{t}_{0}\right)$ and check if $\max _{\mathbf{r}} \operatorname{LSE}^{(0)}(\mathbf{r})<\varepsilon$. If yes, the algorithm is terminated; otherwise continue.

(3) Let $k \leftarrow k+1$ and find $\mathbf{t}_{k}=\arg \left\{\max _{\mathbf{r}}\left[\operatorname{LSE}^{(k-1)}(\mathbf{r})\right]\right\}$.

(4) Apply the FCLS method with the signature matrix $\mathbf{M}^{(k)}=\left[\mathbf{t}_{0} \mathbf{t}_{1} \cdots \mathbf{t}_{k-1}\right]$ to estimate the abundance fraction of $\mathbf{t}_{0}, \mathbf{t}_{1}, \ldots, \mathbf{t}_{k-1}, \hat{\alpha}_{1}^{(k)}(\mathbf{r}), \hat{\alpha}_{2}^{(k)}(\mathbf{r}), \ldots, \hat{\alpha}_{k-1}^{(k)}(\mathbf{r})$.

(5) Find the $k$ th maximum least squares error defined by

$$
\begin{aligned}
& \max _{\mathbf{r}}\left\{\operatorname{LSE}^{(k)}(\mathbf{r})\right\} \\
& =\max _{\mathbf{r}}\left\{\left(\mathbf{r}-\sum_{j=1}^{k-1} \hat{\alpha}_{j}^{(k)} \mathbf{t}_{j}\right)^{T}\left(\mathbf{r}-\sum_{j=1}^{k-1} \hat{\alpha}_{j}^{(k)} \mathbf{t}_{j}\right)\right\} .
\end{aligned}
$$

If $\max _{\mathbf{r}} \mathrm{LSE}^{(k-1)}(\mathbf{r})<\varepsilon$, the algorithm is terminated; otherwise go to step (3).

\section{Unsupervised Spectral Target Finding Algorithms}

When the above-mentioned three unsupervised LS-based algorithms are implemented, a prescribed error $\varepsilon$ which is determined by various applications is required to terminate the algorithms. In general, it is done by visual inspection on a trial-and-error basis and is not practical for our purpose. Therefore, instead of using $\varepsilon$ as a stopping rule, we use the $\mathrm{VD}$ as an alternative rule to determine how many targets are required for our designed LS algorithms to generate.

In order for the proposed LS-based algorithms to be successful, we assume that the most image BKG is characterized by a very large number of un-interesting data sample vectors which can be characterized by 2 nd order statistics as opposed to target pixels which can be captured by highorder statistics due to a small number of target pixels. By virtue of this assumption we can consider two sets of data for processing. One is the original data and the other is the sphered data which has the mean and covariance removed from the original data for consideration. We then apply the three unsupervised LS-based algorithms to these two data sets to extract 2nd order BKG pixels as well as highorder target pixels. However, if a sample pixel show strong signal statistics in both original and sphered data sets, it is considered as a target pixel and can be removed from the BKG category.

A detailed implementation of an LS-based unsupervised spectral target finding algorithm (USTFA) can be briefly described as follows where the LS-based unsupervised algorithm used in the USTFA can be one of the three LS unsupervised algorithms described in Section 2. 


\section{LS-Based Unsupervised Spectral Target Finding Algorithm.}

(1) Find the VD for the image data to determine the number of targets required to be generated, $n_{\mathrm{VD}}$.

(2) Apply an LS-based algorithm to the original image data and find $n_{\mathrm{VD}}$ BKG pixels $S^{\mathrm{BKG}}=\left\{\mathbf{b}_{j}^{\mathrm{LS}}\right\}_{j=1}^{n_{\mathrm{VD}}}$.

(3) Apply the LS-based algorithm to the sphered data and find $n_{\mathrm{VD}}$ target pixels, $S^{\text {target }}=\left\{\mathbf{t}_{j}^{\mathrm{LS}}\right\}_{j=1}^{n_{\mathrm{VD}}}$.

(4) Since there may be some pixels in $S^{\mathrm{BKG}}$ whose spectra are very close to those also showing up in $\mathrm{S}^{\text {target }}$, a spectral measure such as SAM [2] is applied to extract these pixels which will be removed from $S^{\mathrm{BKG}}$. Let the resulting BKG sample set be denoted by $\widetilde{\mathrm{S}}^{\mathrm{BKG}}=\left\{\tilde{\mathbf{b}}_{i}^{\mathrm{LS}}\right\}_{i=1}^{n_{\mathrm{BKG}}}$ where $n_{\mathrm{BKG}}$ is the total number of remaining $\mathrm{BKG}$ pixels in $S^{\mathrm{BKG}}$ after the pixels in $S^{\text {target }} \cap S^{\mathrm{BKG}}$ are removed.

(5) Form a signature matrix $\mathbf{M}$ by merging $\widetilde{S}^{\mathrm{BKG}}$ and $S^{\text {target }}$, that is, finding pixels in $\left\{\tilde{\mathbf{b}}_{i}^{\mathrm{LS}}\right\}_{i=1}^{n_{\mathrm{BKG}}} \cup\left\{\mathbf{t}_{j}^{\mathrm{LS}}\right\}_{j=1}^{n_{\mathrm{VD}}}$. It should be noted that the number of pixels in $\mathbf{M}$ is between $n_{\mathrm{VD}}$ and $2 n_{\mathrm{VD}}$, that is, $n_{\mathrm{VD}} \leq n_{\mathrm{VD}}+n_{\mathrm{BKG}} \leq$ $2 n_{\mathrm{VD}}$.

(6) Apply an LSU method such as abundance-unconstrained classifier LSOSP, abundance Non-negativity constrained classifier NCLS and abundance fully constrained classifier FCLS to perform mixed pixel classification where only the target pixels in $S^{\text {target }}$ will be classified by their corresponding abundance fractions while the target pixels in $\widetilde{\mathrm{S}}^{\mathrm{BKG}}=\left\{\tilde{\mathbf{b}}_{i}^{\mathrm{LS} S}\right\}_{i=1}^{n_{\mathrm{BKG}}}$ will be used for BKG suppression. It should be noted that in order for an LSU to perform purepixel classification we need a value to threshold the LSU-estimated abundance fractions of each of targets for making hard decisions. In this case, finding an appropriate threshold value is generally very challenging. In our experiments conducted in this paper, only LSU is performed to produce abundance fraction estimates for target pixels. So, when a specific LS-based algorithm is used, the superscript "LS" in the above algorithm will be replaced with this particular algorithm. For example, if ATGP is used for the USTFA, it is then called ATGP-USTFA.

\section{Synthetic Image Simulated Scenarios}

The success of the three proposed LS-based algorithms in the unsupervised target analysis hinges on two made hypotheses, (1) targets of interests can be characterized by their spectral statistics, 2nd order targets corresponding to BKG pixels and high-order targets assumed to be desired targets; (2) the VD can be used to estimate the number of targets of interest present in the data. Since neither can be verified by real image scenes where obtaining full scene ground truth is impossible, this section presents two scenarios using a set of controllable parameters to simulate synthetic images via a real Cuprite image data shown in Figure 1 which is available at the USGS

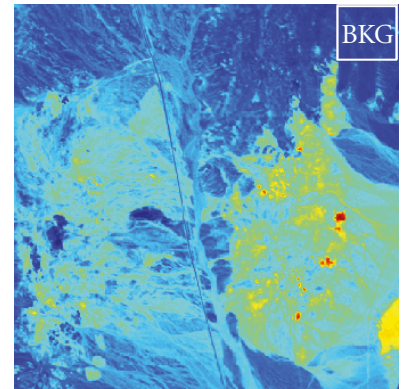

(a)

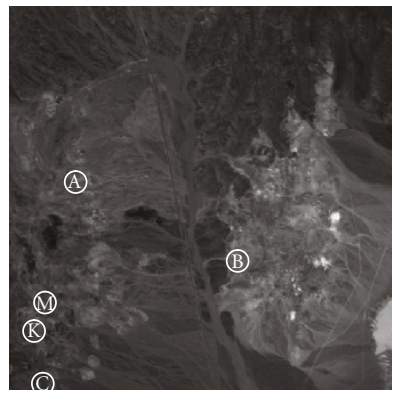

(b)
FIgURE 1: (a) Cuprite AVIRIS image scene, (b) spatial positions of five pure pixels corresponding to minerals: alunite (A), buddingtonite (B), calcite (C), kaolinite (K), and muscovite (M).

website [11]. This scene is a 224-band image with size of $350 \times 350$ pixels and was collected over the Cuprite mining site, Nevada, in 1997. It is well understood mineralogically. As a result, a total of 189 bands were used for experiments where bands $1-3,105-115$, and 150-170 have been removed prior to the analysis due to water absorption and low SNR in those bands. Although there are more than five minerals in the data set, the ground truth available for this region only provides the locations of the pure pixels: Alunite (A), Buddingtonite (B), Calcite (C), Kaolinite (K), and Muscovite (M). The locations of these five pure minerals are labeled by $\mathrm{A}, \mathrm{B}, \mathrm{C}, \mathrm{K}$, and $\mathrm{M}$, respectively, and shown in Figure 1 . Available from the image scene is a set of reflectance spectra shown in Figure 2 which will be used to simulate synthetic images. An area marked by "BKG" at the upper right corner of Figure 1(a) was selected to find its sample mean, that is, the average of all pixel vectors within the area "BKG", denoted by $\mathbf{b}$ to be used to simulate the BKG for image scene in Figure 3 also plotted in Figure 2. The reason for this BKG selection is empirical since the selected area "BKG" seemed more homogeneous than other regions. Nevertheless, other areas can be also selected for the same purpose.

As we can see from the spectral profiles in Figure 2, the Muscovite is the most spectrally distinct signature among all the five signatures and the signature of the Calcite is the most similar to the BKG signature. These two particular signatures will have significant impact on data analysis as demonstrated in the following experiments.

The synthetic image to be simulated for experiments has a size of $200 \times 200$ pixel vectors with 25 panels of various sizes which are arranged in a $5 \times 5$ matrix and located at the center of the scene shown in Figure 3(a).

The 25 panels in Figure 3(a) were simulated as follows. The five mineral spectral signatures, $\left\{\mathbf{m}_{i}\right\}_{i=1}^{5}$ in Figure 2 are used to simulate these 25 panels where each row of five panels was simulated by the same mineral signature and each column of 5 panels has the same size. Among 25 panels are five $4 \times 4$ pure-pixel panels, $p_{4 \times 4}^{i}$ for $i=1, \ldots, 5$ lined up in five rows in the 1 st column and five $2 \times 2$ pure-pixel panels, $p_{2 \times 2}^{i}$ for $i=1, \ldots, 5$ lined up in five rows in the 2nd column for pure pixel classification; the five $2 \times 2$-mixed 


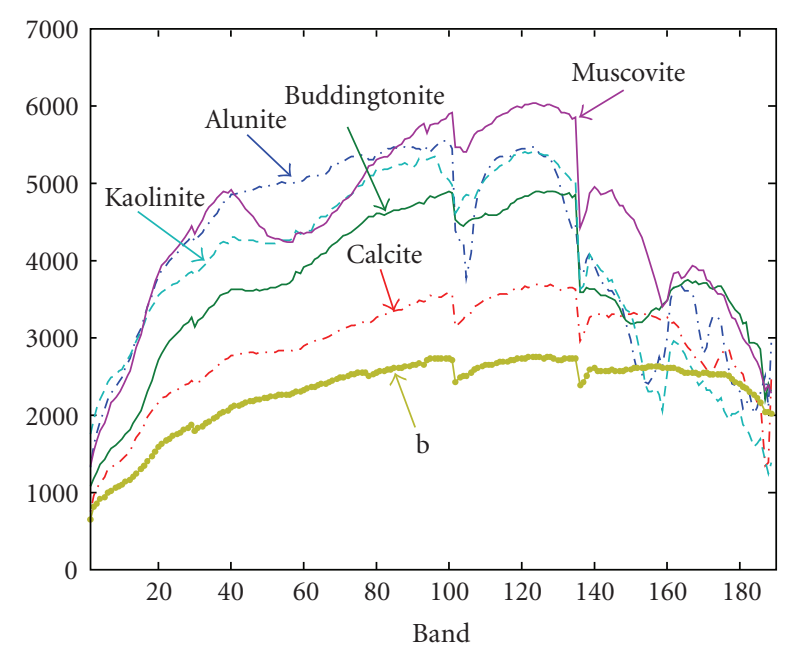

FIGURE 2: Five mineral reflectance spectra and BKG signaturewhich is the average of area BKG in the top right of Figure 1(a).

pixel panels, $\left\{p_{3, j k}^{i}\right\}_{j=1, k=1}^{2,2}$ for $i=1, \ldots, 5$ lined up in five rows in the 3rd column for mixed pixel classification and both the five subpanel pixels, $p_{4,1}^{i}$ for $i=1, \ldots, 5$ lined up in five rows in the 4 th column and the five subpixel panels, $p_{5,1}^{i}$ for $i=1, \ldots, 5$ lined up in five rows in the 5 th column for subpixel classification. The purpose of introducing the 5 panels in the 3rd column and subpanel pixels in the 4th and 5 th columns was designed to conduct a study and analysis on five mineral signatures with different mixing in a pixel and five mineral signatures embedded in single pixels at subpixel scale.

Tables 1 and 2 tabulate the mixing details of the five mineral composition in the 20 mixed pixels in the $3 \mathrm{rd}$ column in Figure 3 and the 5 subpanel pixels with 50\% abundance of mineral signatures in the 4 th column and the 5 subpanel pixels with 25\% abundance of mineral signatures in the 5th columns in Figure 3(a), respectively.

So, in Figure 3(a) there are a total of 130 panel pixels present in the scene, 80 pure panel pixels in the 1st column, 20 pure panel pixels in the 2 nd column, 20 mixed panel pixels in the 3rd column, five 50\%-abundance subpanel pixels in the 4 th column and five $25 \%$-abundaunce subpanel pixels in the 5 th column.

The image BKG was simulated by the signature $\mathbf{b}$ in Figure 2 corrupted by an additive Gaussian noise to achieve a certain signal-to-noise ratio (SNR) which was defined as 50\% signature (i.e., reflectance/radiance) divided by the standard deviation of the noise in [8]. Once target pixels and BKG are simulated, two types of target insertion can be designed to simulate experiments for various applications.

4.1. Target Implantation (TI). The first type of target insertion is referred to as Target Implantation (TI) which inserts the above 130 panel pixels into the image by replacing their corresponding BKG pixels. So, the resulting synthetic image has clean panel pixels implanted in a noisy BKG with an additive Gaussian noise of SNR $=20: 1$ for this scenario as shown in Figure $3(\mathrm{~b})$. The TI is primarily
TABLE 1: Mixed panel pixels in the 3rd column for simulations.

\begin{tabular}{lll}
\hline \multirow{2}{*}{ row 1 } & $p_{3,11}^{1}=0.5 \mathrm{~A}+0.5 \mathrm{~B}$ & $p_{3,12}^{1}=0.5 \mathrm{~A}+0.5 \mathrm{C}$ \\
& $p_{3,21}^{1}=0.5 \mathrm{~A}+0.5 \mathrm{~K}$ & $p_{3,22}^{1}=0.5 \mathrm{~A}+0.5 \mathrm{M}$ \\
\hline \multirow{2}{*}{ row 2 } & $p_{3,11}^{2}=0.5 \mathrm{~A}+0.5 \mathrm{~B}$ & $p_{3,12}^{2}=0.5 \mathrm{~B}+0.5 \mathrm{C}$ \\
& $p_{3,21}^{2}=0.5 \mathrm{~B}+0.5 \mathrm{~K}$ & $p_{3,22}^{2}=0.5 \mathrm{~B}+0.5 \mathrm{M}$ \\
\hline \multirow{2}{*}{ row 3 } & $p_{3,11}^{3}=0.5 \mathrm{~A}+0.5 \mathrm{C}$ & $p_{3,12}^{3}=0.5 \mathrm{~B}+0.5 \mathrm{C}$ \\
& $p_{3,21}^{3}=0.5 \mathrm{C}+0.5 \mathrm{~K}$ & $p_{3,22}^{3}=0.5 \mathrm{C}+0.5 \mathrm{M}$ \\
\hline \multirow{2}{*}{ row 4 } & $p_{3,11}^{4}=0.5 \mathrm{~A}+0.5 \mathrm{~K}$ & $p_{3,12}^{4}=0.5 \mathrm{~B}+0.5 \mathrm{~K}$ \\
& $p_{3,21}^{4}=0.5 \mathrm{C}+0.5 \mathrm{~K}$ & $p_{3,22}^{4}=0.5 \mathrm{~K}+0.5 \mathrm{M}$ \\
\hline \multirow{2}{*}{ row 5 } & $p_{3,11}^{5}=0.5 \mathrm{~A}+0.5 \mathrm{M}$ & $p_{3,12}^{5}=0.5 \mathrm{~B}+0.5 \mathrm{M}$ \\
& $p_{3,21}^{5}=0.5 \mathrm{C}+0.5 \mathrm{M}$ & $p_{3,22}^{5}=0.5 \mathrm{~K}+0.5 \mathrm{M}$ \\
\hline
\end{tabular}

TABLe 2: Subpanel pixels in the 4th and 5th columns for simulations.

\begin{tabular}{ccc}
\hline & $\begin{array}{c}50 \% \text { subpixel panels } \\
\text { in 4th column }\end{array}$ & $\begin{array}{c}25 \% \text { subpixel panels } \\
\text { in } 5 \text { th column }\end{array}$ \\
\hline row 1 & $p_{4,1}^{1}=0.5 \mathrm{~A}+0.5 \mathbf{b}$ & $p_{5,1}^{1}=0.25 \mathrm{~A}+0.75 \mathbf{b}$ \\
row 2 & $p_{4,1}^{2}=0.5 \mathrm{~B}+0.5 \mathbf{b}$ & $p_{5,1}^{2}=0.25 \mathrm{~B}+0.75 \mathbf{b}$ \\
row 3 & $p_{4,1}^{3}=0.5 \mathrm{C}+0.5 \mathbf{b}$ & $p_{5,1}^{3}=0.25 \mathrm{C}+0.75 \mathbf{b}$ \\
row 4 & $p_{4,1}^{4}=0.5 \mathrm{~K}+0.5 \mathbf{b}$ & $p_{5,1}^{4}=0.25 \mathrm{~K}+0.75 \mathbf{b}$ \\
row 5 & $p_{4,1}^{5}=0.5 \mathrm{M}+0.5 \mathbf{b}$ & $p_{5,1}^{5}=0.25 \mathrm{M}+0.75 \mathbf{b}$ \\
\hline
\end{tabular}

designed to simulate scenarios with pure pixels implanted as pure signatures to represent endmembers to evaluate the performance of endmember extraction.

4.2. Target Embeddedness (TE). The second type of target insertion is referred to as Target Embeddedness (TE) which is the same as the TI described above except the way the panel pixels were inserted. The BKG pixels were not removed to accommodate the inserted panel pixels as they were done in TI but were rather superimposed with the inserted panel pixels. So, in this case, the resulting synthetic image shown in Figure 3(c) has clean panel pixels embedded in a noisy BKG. The TE is particularly designed to simulate signal detection models [12] with two hypotheses, null hypothesis corresponding to noise and BKG and the alternative hypothesis specify the embedded target pixels. Under this circumstance, the abundances of the pixels containing inserted targets were not normalized to one in which case the abundance sum to one constraint imposed on FCLS was violated. Nevertheless, it is worth noting that the TE scenario can be also used for endmember extraction to test if an endmember extraction algorithm to be able to extract most purest pixels in case there are no pure pixels present in the data.

Two remarks on scenarios TI and TE are noteworthy.

(1) From Figure 3, one may argue that it is so obvious by visual inspection that most panel pixels in Figure 3 are visible. This may lead to a belief that these two scenarios may not be useful or appropriate. The truth is that what we see from images is generally not what we will expect. Specifically, what we see is only qualitative and not quantitative, a task that a computer algorithm can do well while human being cannot. 


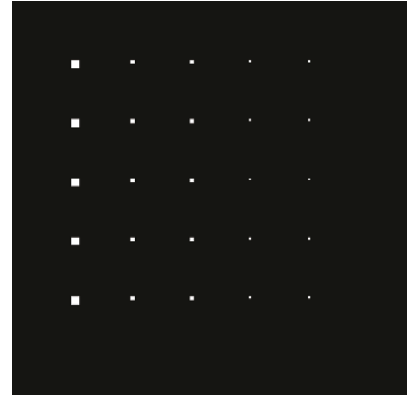

(a) 25 simulated panels

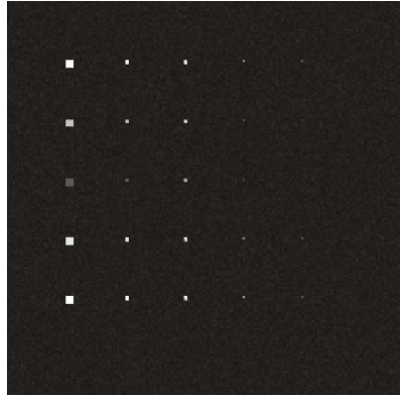

(b) Scenario TI

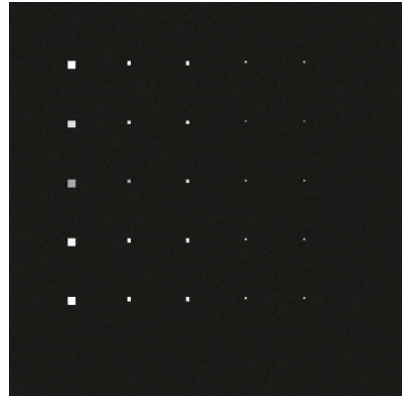

(c) Scenario TE

FIgure 3: 25 simulated panels according to Tables 1 and 2 and two scenarios for target insertion, TI and TE.

This is exactly what we need these scenarios to show that can an algorithm accomplish what human eyes can or do better? Unfortunately, on many occasions misconception of human inspection usually mislead to something incorrect. This phenomenon will be demonstrated in some experiments conducted in Section 5 .

(2) In hyperspectral imagery noise is generally nonGaussian. This is mainly due to the fact that many unknown subtle substances such as clutters, interferers uncovered by hyperspectral imaging sensors are actually interference and not noise, in which case these unwanted interferers should be considered as structure noise to represent bias instead of random noise. If all such unknown substances are absent in the image data which is the case of these two scenarios, it leaves only random noise. Under this circumstance, the Gaussian noise is most appropriate, which is exactly the case that it is assumed in communications. In light of this interpretation, it is reasonable to simulate Gaussian for the scenarios TI and TE because the simulated image BKG is clean.

\section{Synthetic Image Experiments}

This section presents three applications, endmember extraction, unsupervised target detection, and LSU-based target classification to show that each application requires a different level of a posteriori information to perform target analysis. Among these applications the endmember extraction is one that needs the least information with only the number of endmembers, $p$ required to be known. The value of the $p$ can be determined by the VD. To the contrary, the LSU-based target classification requires the most information including a posteriori information to be used to form the linear mixture model for LSU.

Assume that no prior knowledge about the scenarios TI and TE is provided. In both scenarios the VD-estimated value, $n_{\mathrm{VD}}$ was 6 as long as the false alarm probability $P_{F} \leq 10^{-1}$. Therefore, $n_{\mathrm{VD}}=6$ was used for the value of $p$ throughout the experiments conduced in this section. Figures $4(\mathrm{a})-4(\mathrm{~d})$ and $5(\mathrm{a})-5(\mathrm{~d})$ show the target pixels in TI and TE found by the 3 LS-based methods, referred to as ATGP-USTFA, UNCLS-USTFA, and UFCLS-USTFA using $n_{\mathrm{VD}}=6$ where (a) the 2 nd order BKG pixels obtained by applying an LS-based unsupervised algorithm to the original data; (b) high-order target pixels obatined by applying the same algorithm to the sphered data; (c) the remaining BKG pixels in (a) after removing BKG pixels which were also found as target pixels; (d) total desired pixels obtained by combining the pixels in (b) and (c). According to the results obtained for the scenarios TI and TE in Figures 4 and 5, target pixels and BKG pixels overlapped the total number of pixels of interest have been found to be either 6 or 7 with five pure panel pixels plus one or two pixels corresponding to either subpanel pixels or BKG pixels. These found target and BKG pixels can be used a posteriori target information for further follow-up various tasks in image analysis.

5.1. Endmember Extraction. In this section, two well-known endmember extraction algorithms, PPI [13] and N-finder algorithm (N-FINDR) [14] were implemented for endmember extraction. According to the ground truth used to simulate the two scenarios TI and TE, the $p$ for TI and TE are 5 and 0 , respectively, but the VD estimated for both scenarios TI and TE was $6, n_{\mathrm{VD}}=6$. Since both require data dimensionality reduction prior to endmember extraction, the maximum noise fraction MNF [15] was to reduce data dimensionality to 5 and 6 , that is, the number of dimensions needed to be retained for analysis, $q=5$ and 6. Figure 6(a) shows endmember extraction results by the PPI using 200 skewers by letting $q=5$ and 6 for both scenarios TI and TE. Due to the fact that the PPI does not have prior knowledge about the value of $p$ and provides no guideline to select endmembers, all the data sample vectors with their PPI counts greater than 0 were extracted for endmember extraction to ensure that no pure panel pixels were left out. But, it does not mean that all the data sample vectors were endmembers. As shown in Figure 6(a) all the 100 panel pixels in the first 2 columns were among many hundreds of pixels extracted by the PPI in the TI and TE scenarios. As a matter of fact, some pure panel pixels in these experiments had their PPI counts with the smallest value 1 . This implies that it is generally not true that a data sample vector with a higher PPI count is a more likely endmember. In this case, the PPI required human intervention to choose an appropriate value 


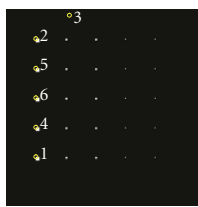

(a) 6 BKG pixels

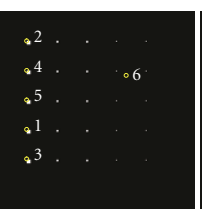

(b) 6 target pixels

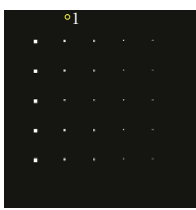

(c) 1 BKG pixel

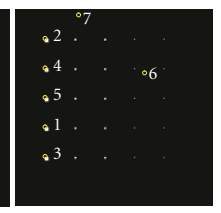

(d) 7 pixels in $(b+c)$

(i) ATGP-USTFA and UNCLS-USTFA

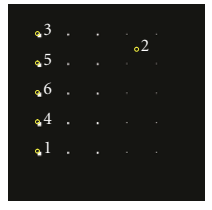

(a) 6 BKG pixels

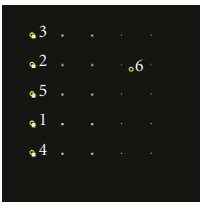

(b) 6 target pixels

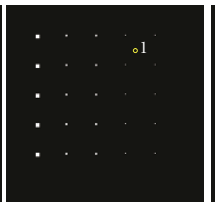

(ii) UFCLS-USTFA

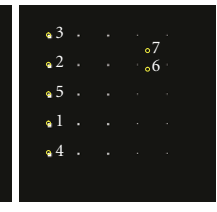

(d) 7 pixels in $(b+c)$

FigURE 4: Target pixels extracted by three unsupervised algorithms ATGP-USTFA, UNCLS-USTFA, and UFCLS-USTFA for scenario TI.

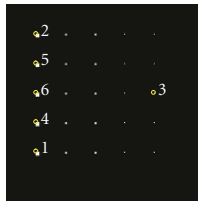

(a) 6 BKG pixels

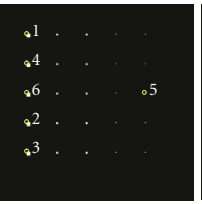

(b) 6 target pixels

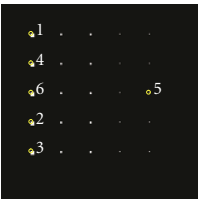

(d) 6 pixels in $(b+c)$ (c) 0 BKG pixel UNCLS-USTFA

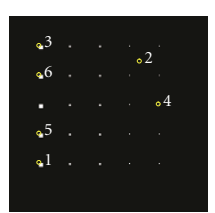

(a) 6 BKG pixels

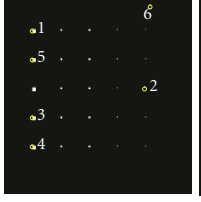

(b) 6 target pixels

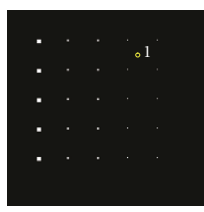

(c) 1 BKG pixel

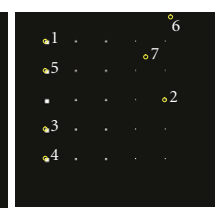

(d) 7 pixels in $(b+c)$

(ii) UFCLS-USTFA

FIGURE 5: Target pixels extracted by three unsupervised algorithms ATGP-USTFA, UNCLS-USTFA, and UFCLS-USTFA for scenario TE.
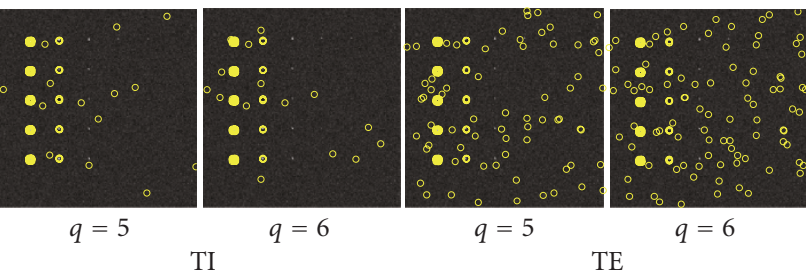

(a) PPI using 200 skewers for TI and TE

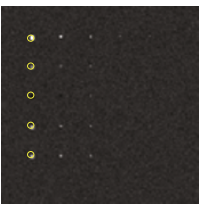

$p=5$

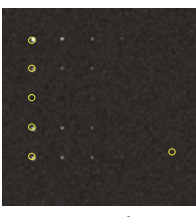

$p=6$

TI

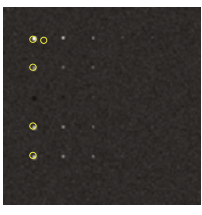

$p=5$ TE (b) Endmembers extracted by N-FINDR for TI and TE

FIGURE 6: Endmember extraction results by PPI and N-FINDR for TI and TE.

to threshold PPI counts to find desired endmembers despite that it does not need to know $p$. This shows that the human manipulation is a key factor to make the PPI successful and effective.

Unlike the PPI the N-FINDR did require the knowledge of the $p$ in which case we assumed that $p=n_{\mathrm{VD}}=5$ and 6 for both scenarios. Figure 6(b) shows the endmember extraction results where the N-FINDR successfully extracted the first panel pixels in each of five different rows that corresponded to the five distinct mineral signatures as endmembers for TI with $p=5$ and 6 . However, it is interesting to note that this was not true for TE with $p=5$ where it missed the panel pixels in the 3rd row. In order to extract a panel pixel in the 3rd row the $p$ must be assumed to be at least 6 as shown in the experiment. This was due to the fact that there were no endmembers present in the scenario TE and the $\mathrm{N}$ FINDR tried to extract the most purest panel pixels from the data. When the value of $p$ was set to low such as 5 , the NFINDR extracted a mixed BKG pixel instead of panel pixels in the 3 rd row as an endmember in which case the BKG pixel exhibited more purity than the panel pixels in the 3rd row. This also explained that the endmembers did not necessarily have higher PPI counts in Figure 6(a). This phenomenon was also well demonstrated in Figure 5(i) where the first panel pixel in the 3rd row was extracted as the sixth target pixels. If we further compare the results in Figure 6 to those in Figure 4, it is clear that the three LS-based algorithms performed as if they were endmember extraction algorithms which were able to extract all the five endmembers from both the original data and the sphered data for TI as the PPI and N-FINDR did in Figures 6(a) and 6(b). However, for the TE experiments in Figure 5, only the UFCLS-USTFA missed one endmember in the 3rd row due to the fact that the TE did not satisfy the abundance sum-to-one constraint and the UFCLS was a fully abundance-constrained algorithm. Nevertheless, the three LS-based algorithms were also able to extract pixels that corresponded to most purest signatures. The experiments demonstrated by Figures 4 and 5 showed that the three LS-based algorithms can be also used for the purpose of endmember extraction provided that $n_{\mathrm{VD}}$ is set to $p$ in which case some of extracted targets pixels may not be endmembers, particularly, for the TE scenario. This makes sense since a spectrally distinct signature is not necessarily an endmember and $n_{\mathrm{VD}}$ is generally greater than or equal to $p$. In the scenarios of TI and TE, the $p$ is supposed to be 5 and 0 , respectively. The reason that $n_{\mathrm{VD}}=6>p=5$ is because the BKG spectral signature $\mathbf{b}$ in Figure 2 used to simulate the image BKG is very distinct from the other five mineral signature in which case it must be considered as a signature even it is a mixed signature. However, if we use a BKG signature equally mixed by the five mineral signatures to replace the $\mathbf{b}$ in Figure 2 to simulate the image BKG, the $n_{\mathrm{VD}}$ 


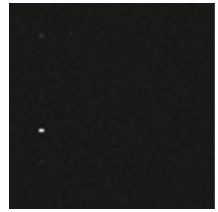

Panels in row 4

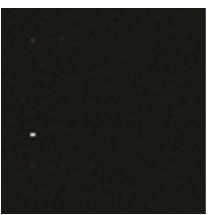

Panels in row 4

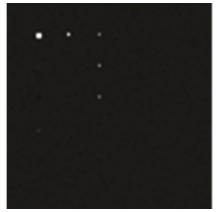

Panels in row 1

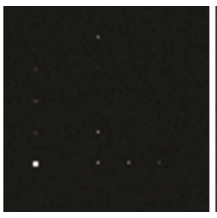

Panels in row 5

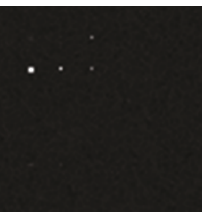

Panels in row 2

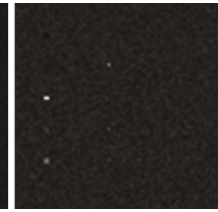

Panels in row 3

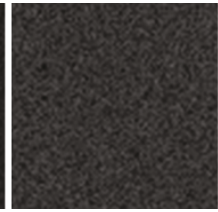

BKG

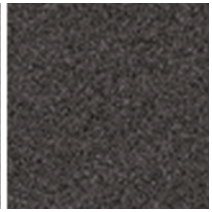

BKG

(a) CEM in conjunction with ATGP-USTFA and with UNCLS-USTFA

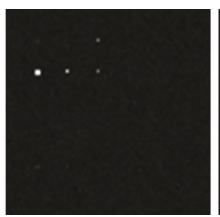

Panels in row 2

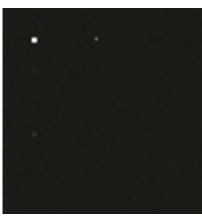

Panels in row 1

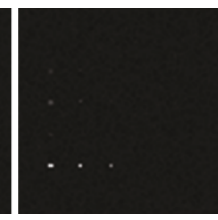

Panels in row 5

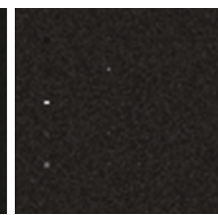

Panels in row 3

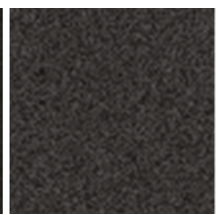

BKG

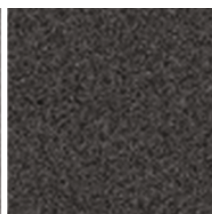

BKG

(b) CEM in conjunction with UFCLS-USTFA

FIgURE 7: CEM detection results for TI.

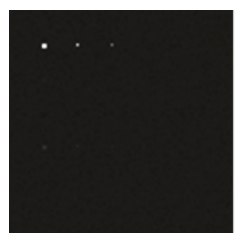

Panels in row 1

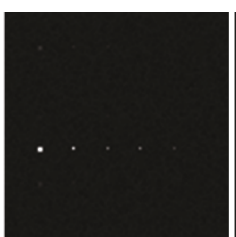

Panels in row 4

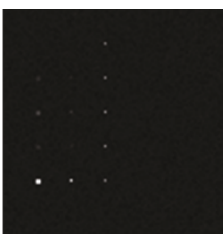

Panels in row 5

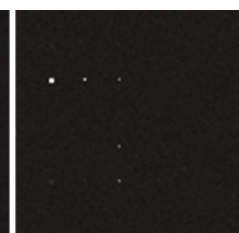

Panels in row 2

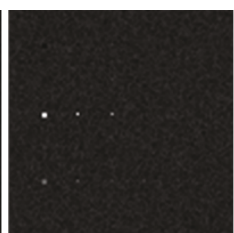

Panels in row 3

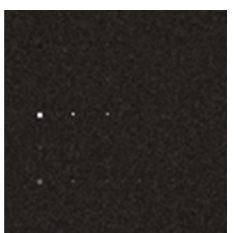

Panels in row 3

(a) CEM in conjunction with ATGP-USTFA and with UNCLS-USTFA

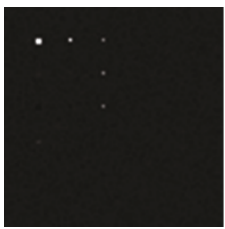

Panels in row 1

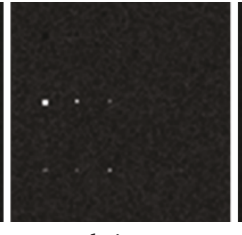

Panels in row 3

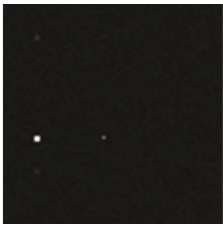

Panels in row 4

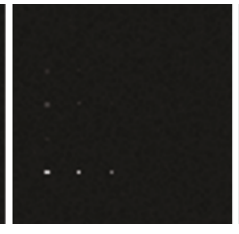

Panels in row 5

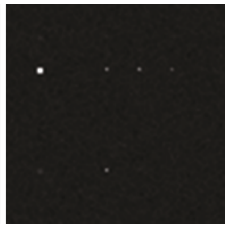

Panels in row 2

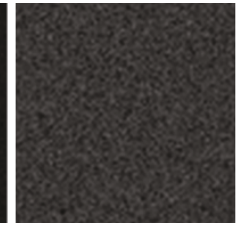

BKG

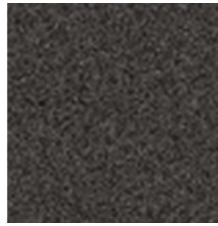

$\mathrm{BKG}$

(b) CEM in conjunction with UFCLS-USTFA

FIGURE 8: CEM detection results for TE.

turns out to be 5 which is exactly the same as $p=5$. Since the result was reported in [16] with more details, it is not included here.

\subsection{Unsupervised Target Detection. Unlike endmember ex-} traction the unsupervised target detection extracts targets regardless of whether or not they are endmembers. As defined previously, the targets of interest to be considered in this paper are specified by their statistical properties in spectral characterization and were extracted in Figures $4(\mathrm{~d})$ and 5(d) by three LS-based algorithms. Then these found targets of interest were further as a posteriori target information to perform unsupervised target detection by the constrained energy minimization (CEM) developed in [17] where Figures 7 and 8 show their CEM-detection results for TI and TE, respectively.

As shown in Figures 7 and 8, the unsupervised CEMbased target detection performed well using each of the found targets of interest as a desired target signature.
Comparing the result in Figure 8 to that in Figure 7 it seemed that the CEM performed better in TE than in TI due to the fact that the target panels were superimposed atop the BKG pixels.

One comment on differentiating the above unsupervised target detection from anomaly detection is noteworthy. While the former requires a posteriori target knowledge to detect specific targets, the latter performs target detection without any target knowledge whatsoever. In this case, it does not know what targets it detects. The following simple example provides a clue of how controversial this issue is.

Figures $9(\mathrm{a})$ and 9 (b) shows a set of the same various target panels with four different sizes implanted in two uniform image BKGs with sizes of $64 \times 64$ pixel vectors and $200 \times 200$ pixel vectors, respectively, where the 5 panels in the 1st column are size of $6 \times 6$ pixel vectors, the 5 panels in the 2 nd column are size of $3 \times 3$ pixel vectors, the 5 panels in the 3 rd column are size of $2 \times 2$ pixel vectors, and then the 5 panels in the 4 th column are size of $1 \times 1$ pixel vectors. 


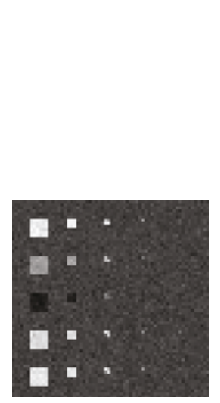

(a) Image of $64 \times$ 64 pixels

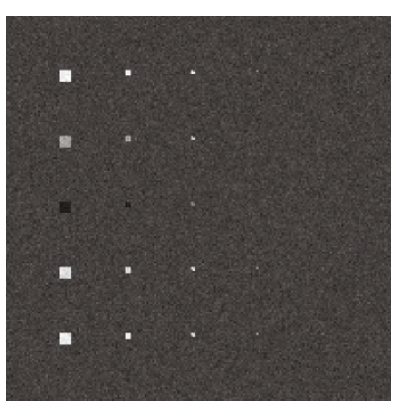

(b) Image of $200 \times 200$ pixels

Figure 9: Target panels with four various sizes implanted in two uniform image BKGs with sizes of $64 \times 64$ pixel vectors and $200 \times 200$ pixel vectors.

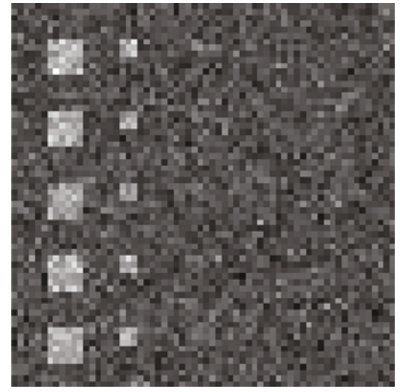

(a) Image of $64 \times 64$ pixels

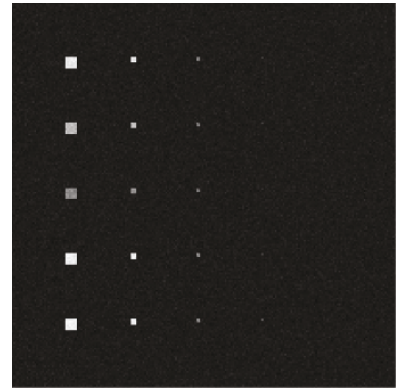

(b) Image of $200 \times 200$ pixels
FIGURE 10: Results of operating RXD on images in Figures 9(a) and 9(b).

The target panels in Figures 9(a) and 9(b) were implanted by replacing the BKG pixels with target panel pixels as the TI scenario does. Figures 10 (a) and 10(b) shows the results of operating a widely used anomaly detector developed by Reed and $\mathrm{Yu}$ [18], called RX detector (RXD) on the two images in Figures 9(a) and 9(b), respectively, where RXD has struggled with finding panel pixels in 3rd-5th columns in Figure 10(a) and also missed most of subpanel pixels in the 4th and 5 th columns in Figure 10(b). In addition, the results in Figures $10(\mathrm{a})$ and 10 (b) did not discriminate target pixels it detected.

An immediate finding by comparing the results in Figure 10(b) to that in Figure 10(a) leads to an interesting observation: the target panels of sizes $2 \times 2$ and $1 \times 1$ that are detected by the RXD in Figure 10(b) as anomalies now become undetectable and are no longer anomalies in Figure 10(a) where two images in Figures 10(a) and 10(b) are shown in the same size for clear and better visual assessment. Moreover, the target panels of sizes $6 \times 6$ and $3 \times 3$ detected in Figure 10(a) also become smeared and blurred compared to their counterparts in Figure 10(b) which are detected clearly as anomalies. Why does the same RXD produce so different results for the same set of target panels? This simple example sheds light on several issues resulting from the RXD. As shown in [3] the image size had tremendous effect on the RXD performance in which case one target detected as an anomaly in a large image size may not be an anomaly in a smaller image size. Another issue is determination of number of anomalies detected by the RXD. Since the RXD generates real values of all image pixels which can be considered as detected abundance fractions, it requires an appropriate threshold to determine which pixel is anomaly and which pixel is not. A third main issue is well-illustrated in Figure 10 where the RXD cannot discriminate among all pixels it detected. All of these issues present challenges for image analysts. It is interesting to note that our proposed LS-based approach provides solutions to all these three issues.

5.3. Linear Spectral Unmixing for Target Classification. A key to the success in LSU is to find an appropriate signature matrix $\mathbf{M}$ to form a linear mixing model $\mathbf{r}=\mathbf{M} \boldsymbol{\alpha}+\mathbf{n}$ where $\mathbf{r}$ is an image pixel and $\mathbf{n}$ is a model correction term. In the supervised LSU (SLSU), this matrix $\mathbf{M}$ is assumed to be known a priori. However, when it comes to unsupervised LSU (ULSU) the knowledge of the signature matrix $\mathbf{M}$ is not available and must be obtained directly from the data. The unsupervised LS-based target finding algorithm presented in Section 2 provides a means of finding such matrix M. More specifically, let $\left\{\tilde{\mathbf{b}}_{i}^{\mathrm{LS}}\right\}_{i=1}^{n_{\mathrm{BKG}}}$ and $\left\{\mathbf{t}_{j}^{\mathrm{LS}}\right\}_{j=1}^{n_{\mathrm{VD}}}$ be BKG and target signatures found by an LS-based target finding algorithm. Then we can form a desired signature matrix $\mathbf{M}=\left[\mathbf{t}_{1}^{\mathrm{LS}} \mathbf{t}_{2}^{\mathrm{LS}} \cdots \mathbf{t}_{n_{\mathrm{VD}}}^{\mathrm{LS}} \tilde{\mathbf{b}}_{1}^{\mathrm{LS}} \tilde{\mathbf{b}}_{2}^{\mathrm{LS}} \cdots \tilde{\mathbf{b}}_{n_{\mathrm{BKG}}}^{\mathrm{LS}}\right]$ to unmix all image pixels r. Figures 11 and 12 show the unmixed results which classified the entire image into $n V D$ spectral classes via target pixels, $\left\{\mathbf{t}_{j}^{\mathrm{LS}}\right\}_{j=1}^{n_{\mathrm{VD}}}$ found by the USTFA for scenarios TI and TE, respectively where the ATGP, UNCLS, and UFCLS were used as the USTFA and the classification was performed by three linear spectral unmixing methods, Least Squares Orthogonal Subspace Projection (LSOSP), referred to as signature subspace projection (SSP) in [2, pages 144-146] and [19], Non-negativity Constrained Least Squares (NCLS) [6] and Fully Constrained Least Squares (FCLS) [7].

The results in (i), (ii), and (iii) of Figures 11 and 12 were obtained by using LSOSP, NCLS, and FCLS to unmix data samples in the TI and TE scenarios via the signature matrix $\mathbf{M}$ formed by the target pixels found in Figures 4(d) and 5(d), respectively, where target pixels were identified by the ground truth along with their quantification results for comparison. It should be noted that each figure was arranged in the order extracted by the unsupervised target algorithm in Figures $4(d)$ and $5(d)$. Since the whole process is unsupervised we must unmix the data using all target pixels including the BKG pixels. Figure 12 shows the linear unmxing results performed on the scenario TE using the target pixels found in Figure $5(d)$. Due to the use of a subpanel pixel $p_{5,1}^{3}$ in Figure 5(d) as one of the target signatures to unmix TE the resulting abundance fractions for the two subpanel pixels were $100 \%$ in Figure 12(b) by all the three LSU methods compared to the case in Figure 12(a) where the target and BKG pixels found by ATGP and UNCLS were used for unmixing and the two subpanel pixels were unmixed to their correct abundance fractions of $50 \%$ and $25 \%$ Calcite, 

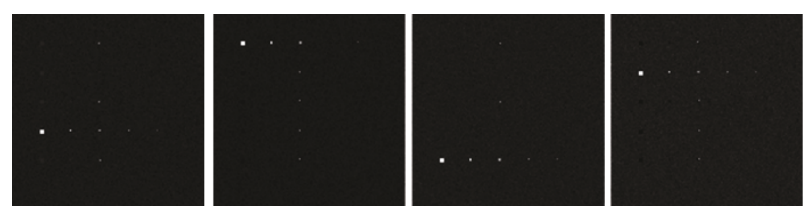

(i) LSOSP
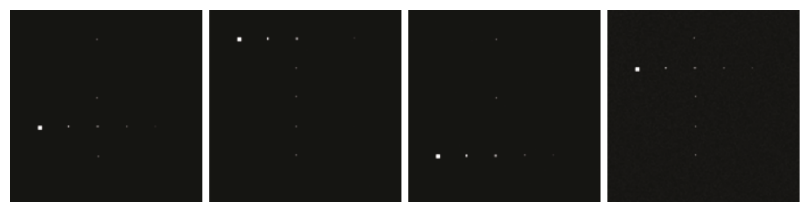

(ii) NCLS

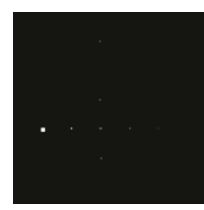

Panels in row 4

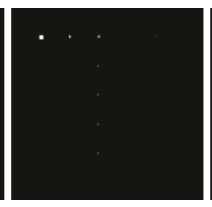

Panels in row

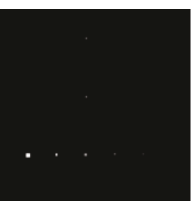

Panels in row 5

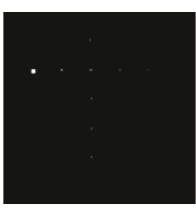

Panels in row 2

(iii) FCLS

(a) ATGP-USTFA and UNCLS-USTFA
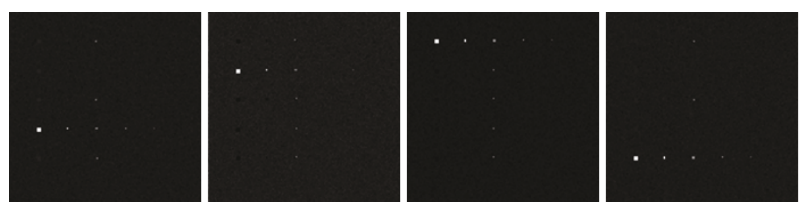

(i) LSOSP
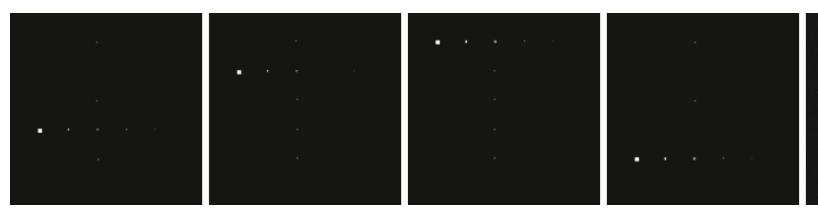

(ii) NCLS

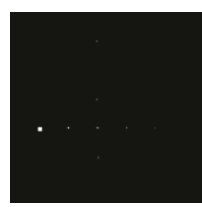

Panels in row 4

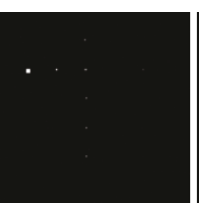

Panels in row 2

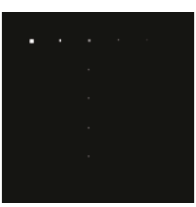

Panels in row 1

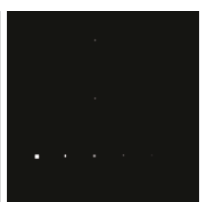

Panels in row 5

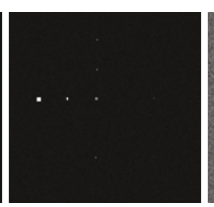

(iii) FCLS
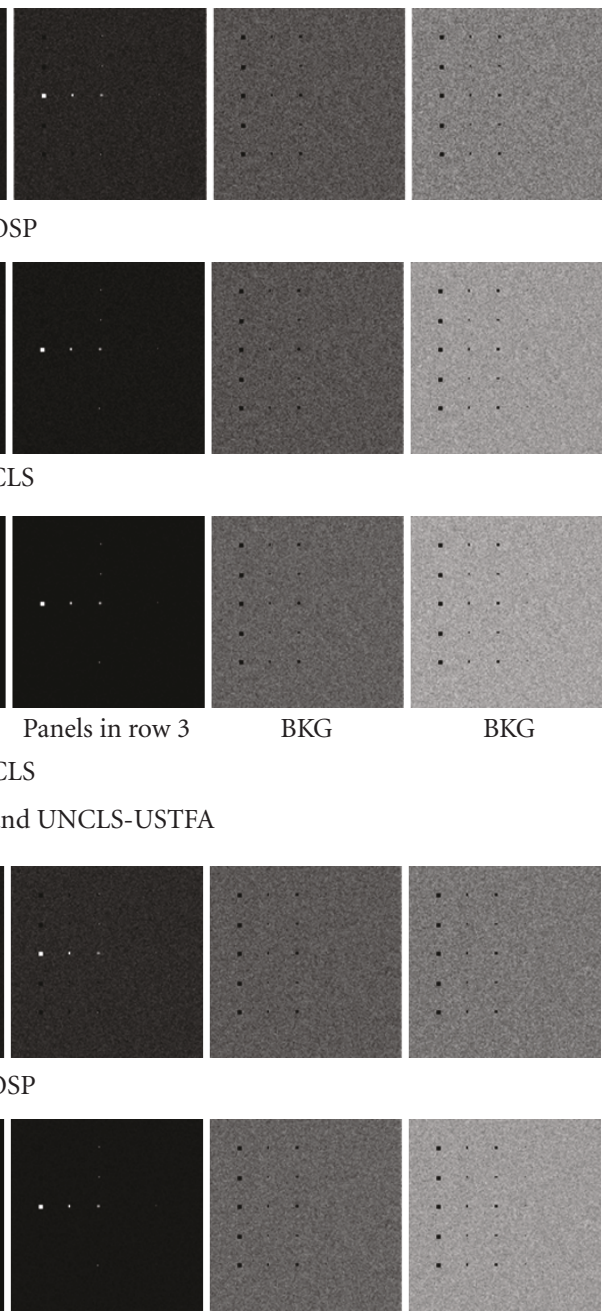

BKG

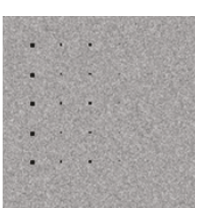

BKG
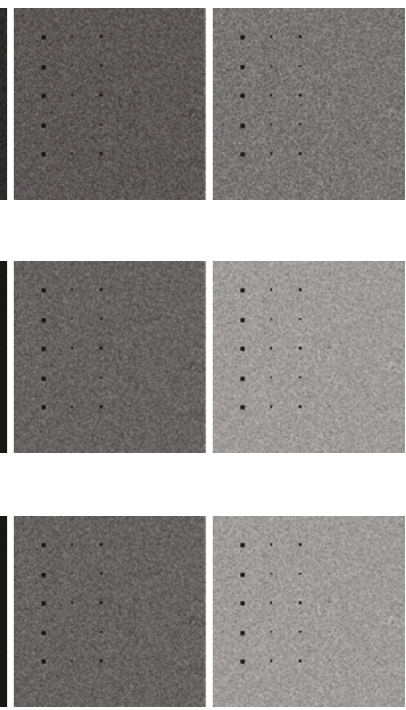

$\mathrm{BKG}$

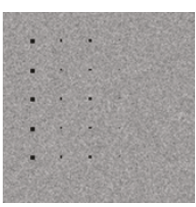

BKG

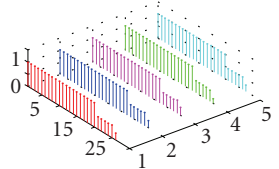

Quantification

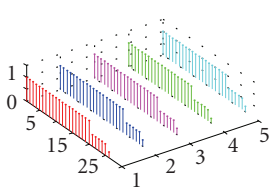

Quantification

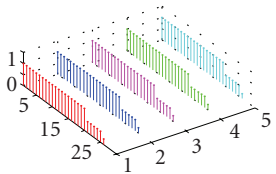

Quantification

(b) UFCLS-USTFA

FIgURE 11: Results of using LSOSP, NCLS, and FCLS to unmix TI via the target pixels found in Figure 4(d).

respectively. Since LSOSP is unconstrained, the 20 pure panel pixels in row 3 were overestimated in Figures 12(b)-12(i) by using the subpanel pixel $p_{5,1}^{3}$ for unmixing.

In order to further investigate this intriguing finding, we conducted experiments to use the five mineral signatures plus the BKG signature plotted in Figure 2 to form the signature matrix $\mathbf{M}$ and then perform SLSU for scenarios TI and TE using the same three LSU methods. Figures 13(a)13(c) and 14(a)-14(c) show their unmixed results for all the 130 panel pixels along with their detected abundance fractions for TI and TE, respectively. Comparing the results in Figures 13 and 14 obtained by SLSU and Figures 11 and 12 obtained by USLU the results were comparable in terms of quantification for both scenarios TI and TE except one case of the TE scenario where FCLS was used to perform SLSU and the resulting abundance fractions for every single panel pixel in row 5 were estimated to be $100 \%$ as opposed to zero for every pixel in rows 1-4 as shown in Figure 14(c). The main reason is that the panel pixels in the TE scenario were added to the BKG pixels such that the abundance fractions of panel pixels and BKG pixels were not summed up to one. However, even though the abundance sum-to-one constraint 

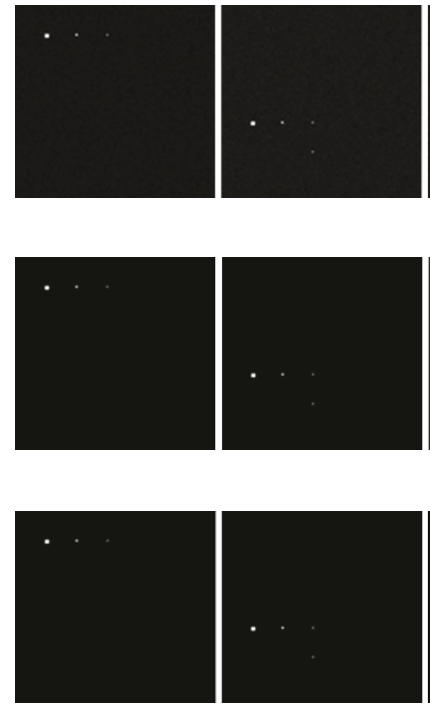

Panels in row 1

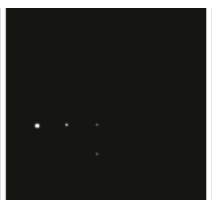

Panels in row 4
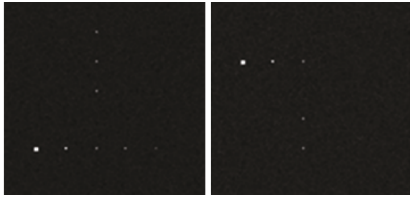

(i) LSOSP
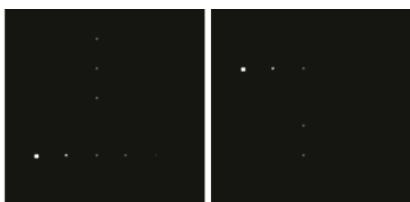

(ii) NCLS

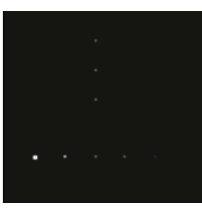

Panels in row 5

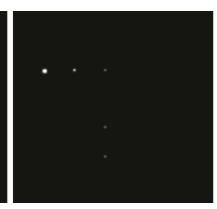

Panels in row 2
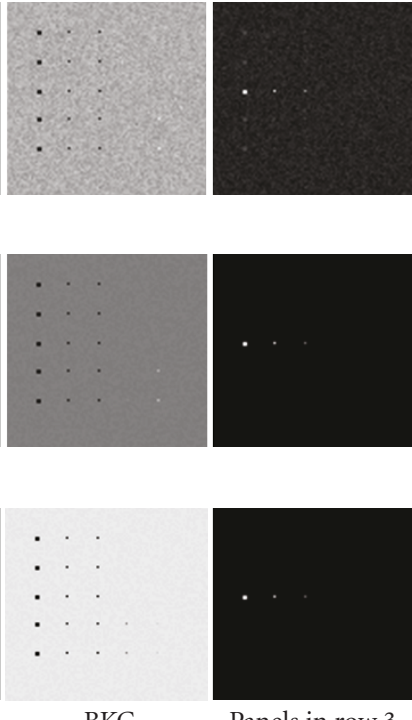

BKG

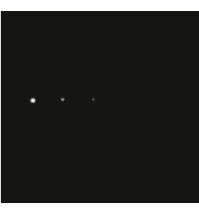

Panels in row 3

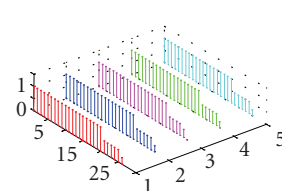

Quantification

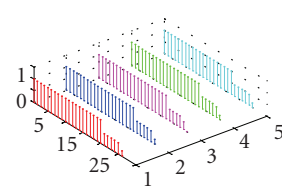

Quantification

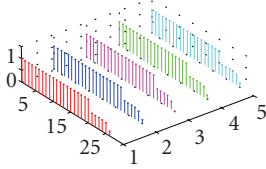

Quantification

(a) ATGP-USTFA and UNCLS-USTFA
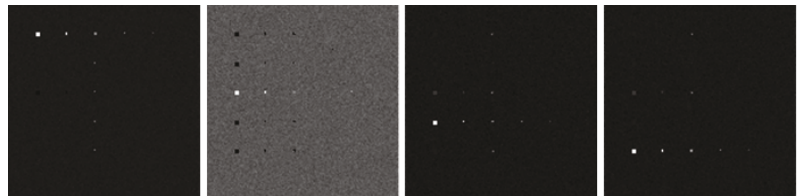

(i) LSOSP
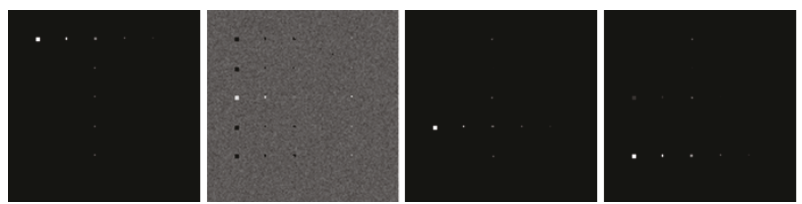

(ii) NCLS

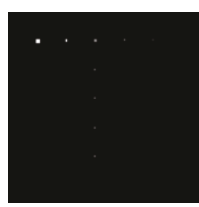

Panels in row 1

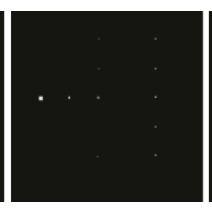

Panels in row 3

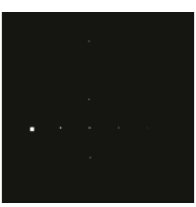

Panels in row 4

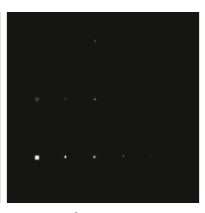

Panels in row 5
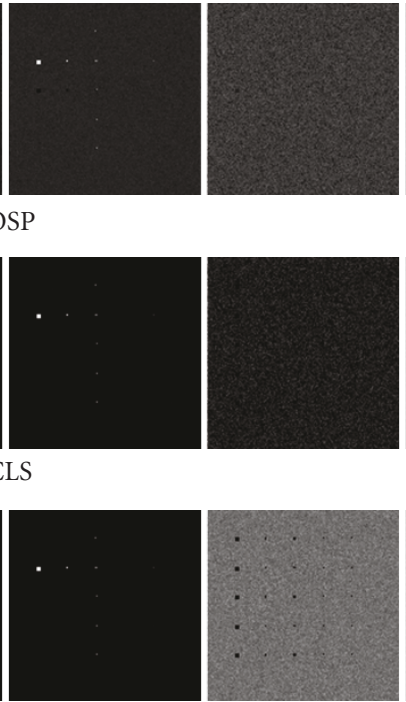

Panels in row 2

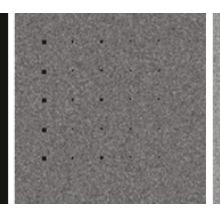

$\mathrm{BKG}$
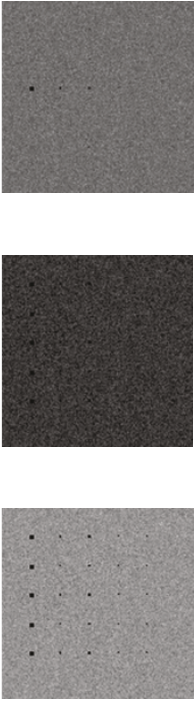

BKG

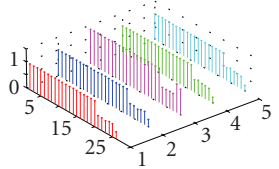

Quantification

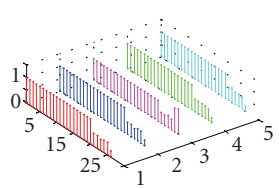

Quantification

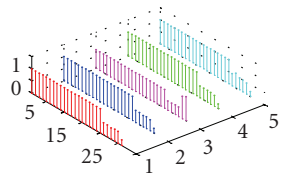

Quantification

(iii) FCLS

(b) UFCLS-USTFA

Figure 12: Results of using LSOSP, NCLS, and FCLS to unmix TE via the target pixels found in Figure 5(d).

assumption is violated in TE, the FCLS still tried to impose the constraint by giving all abundance fractions to the most distinct spectral signature which is Muscovite in this image used to simulate panel pixels in row 5. For this particular case the ULSU was superior to the SLSU because USLU obtains target knowledge directly from the data which may be more realistic and accurate than the prior knowledge used by the SLSU. However, in the scenario TI the panel pixels are implanted into the BKG with the corresponding BKG pixels removed in which case the abundance sum-to-one assumption still holds. As a result, the FCLS performed well regardless of whether the LSU is performed supervised or unsupervised. Since the LSOSP and NCLS did not impose the abundance sum-to-one constraint they performed well for both TI and TE scenarios. These experiments also provide strong evidence of the advantages of using synthetic images because it is nearly impossible to conduct such experiment using real image data where no complete ground truth is available that can be used for quantitative and qualitative data analyses.

By concluding this section two comments are worthwhile. 

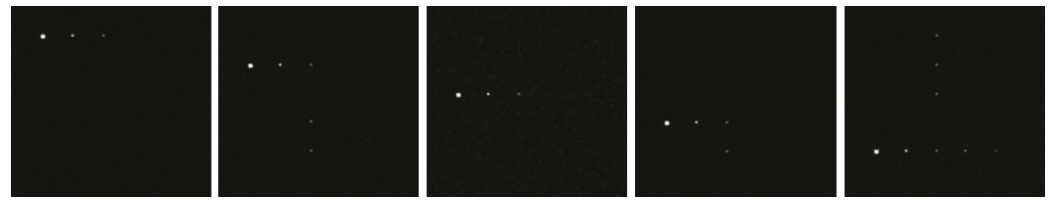

(a) LSOSP
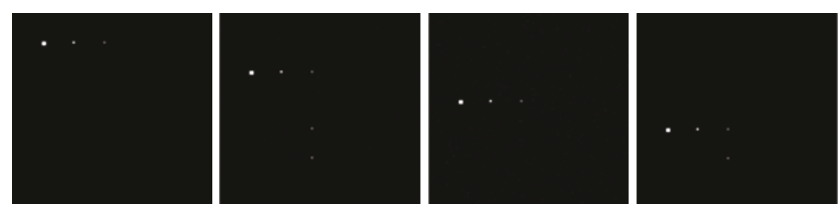

(b) NCLS

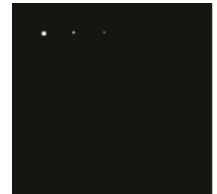

Panels in row 1

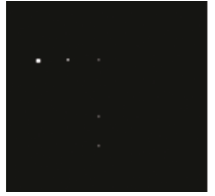

Panels in row 2

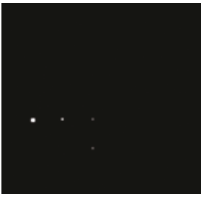

Panels in row 3

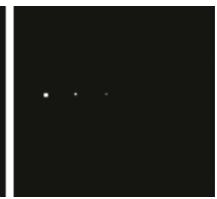

Panels in row 4
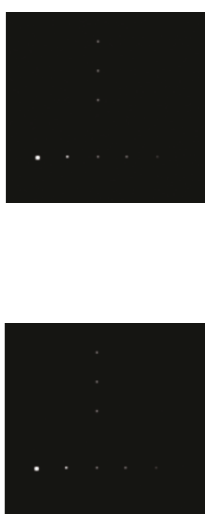

Panels in row 5

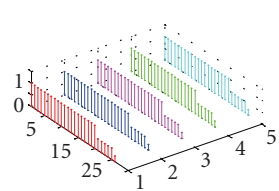

Quantification

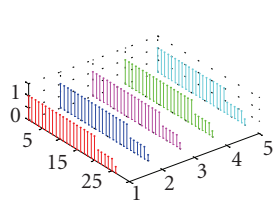

Quantification

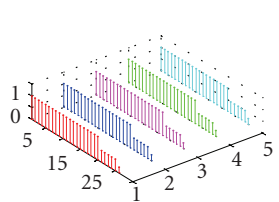

Quantification

(c) FCLS

FIGURE 13: Results using LSOSP, NCLS, and FCLS to unmix TI with assuming signature knowledge in Figure 2.
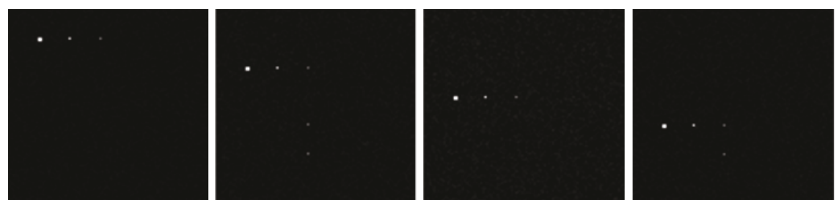

(a) LSOSP
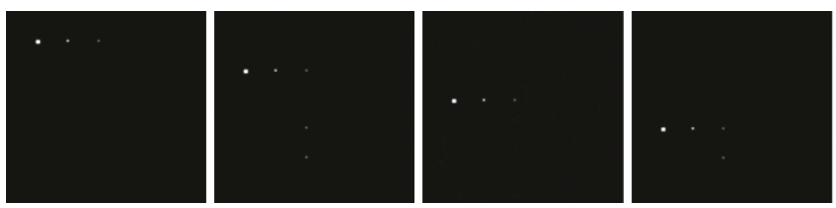

(b) NCLS

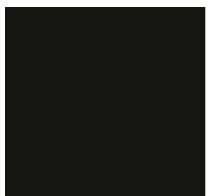

Panels in row 1

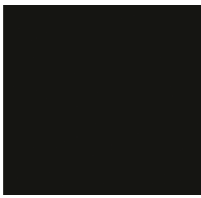

Panels in row 2

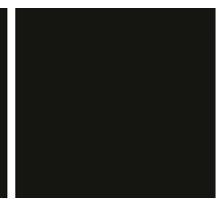

Panels in row 3

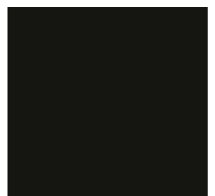

Panels in row 4

FCLS
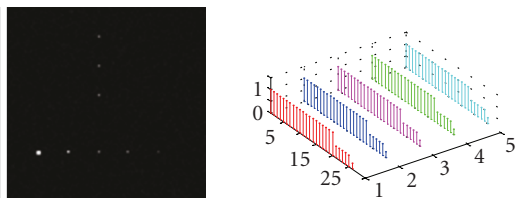

Quantification
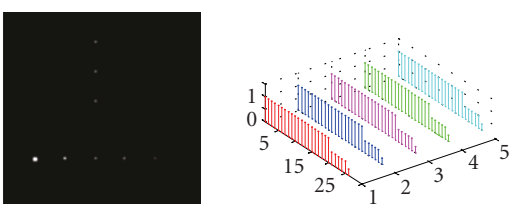

Quantification

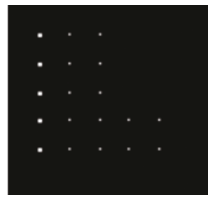

Panels in row 5

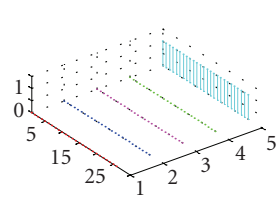

Quantification

FIGURE 14: Results using LSOSP, NCLS, and FCLS to unmix TE with assuming signature knowledge in Figure 2.

(1) Although the two synthetic image scenarios seem simple, the value of the experiments should be appreciated. These scenarios provide an objective validation of any designed algorithm under a fully controllable environment with complete ground truth.
A good example is illustrated by Figure 14(c) where the FCLS completely failed in the scenario TE because the sum-to-one abundance constraint is violated. If it had been applied to real data we would not have known that a fully abundance constrained LSU could 


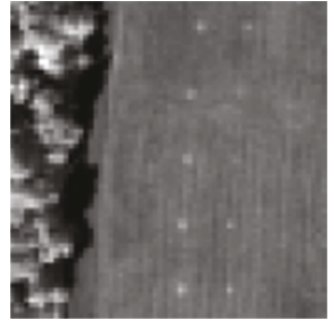

(a)

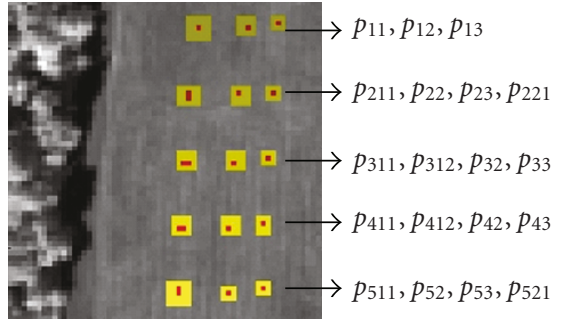

(b)

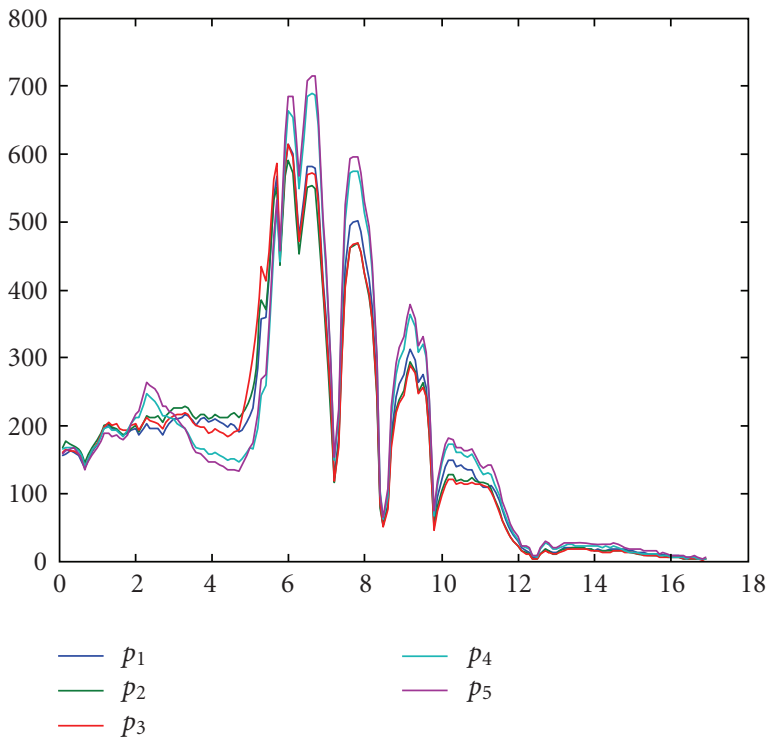

(c)

Figure 15: (a) A HYDICE panel scene which contains 15 panels; (b) Ground truth map of spatial locations of the 15 panels; (c) five panel signatures $p_{1}, p_{2}, p_{3}, p_{4}, p_{5}$.

not be used as a signal detection technique when the linear mixing model was used as a signal/noise detection model in which case a signal is embedded in a pixel corrupted by an additive noise like the scenario TE so that the abundance fractions of the signal and noise were not summed up to one. If an algorithm does not pass the synthetic image experiments, it will be very likely that it may not work in real data.

(2) Due to significantly improved spectral resolution provided by hyperspectral imaging sensors hyperspectral imaging generally performs "target"-pixelbased spectral analysis rather than "class-map/pattern"-based spatial analysis as conduced in traditional image processing. Therefore, BKG pixels are usually not of major interest and no BKG analysis is necessary for hyperspectral imaging. Instead, they are only used for BKG suppression to improve target detection and classification. Because of that the scenarios TI and TE suffice to serve the purpose where only complete knowledge of target panel pixels is required for target analysis and BKG pixels can be made as simple as possible by adding Gaussian noise for suppression.

\section{Real Image Experiments}

In the previous section the synthetic image experiments were used to show the unsupervised target analysis in three applications where the ground truth was used to substantiate the results. In this section, we further conduct real image experiments to demonstrate that unsupervised target analysis is indeed superior to supervised target analysis. The reason for this is that the prior knowledge used by the supervised target analysis generally does not represent true knowledge about the real data because of many unknown factors such as interference, noise, and so forth present in the data that may contaminate the prior knowledge. The required true knowledge must be acquired and obtained directly from the real data itself. Two sets of real data are used for experiments.

6.1. HYDICE Data. The first data is a real HYperspectral Digital Image Collection (HYDICE) scene shown in Figure 15 (a) and has a size of $64 \times 64$ pixel vectors with 15 panels in the scene and the ground truth map in Figure 15(b). It was acquired by 210 spectral bands with a spectral coverage from $0.4 \mu \mathrm{m}$ to $2.5 \mu \mathrm{m}$. Low signal/high 


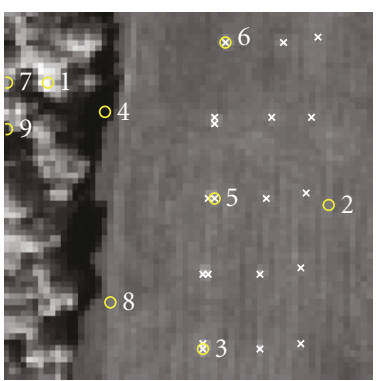

(a)

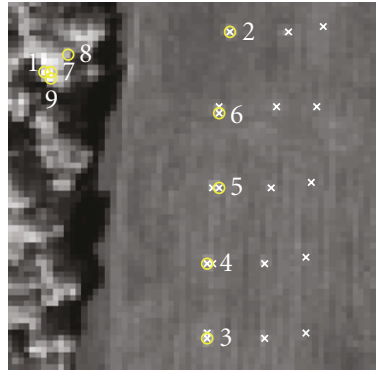

(b)

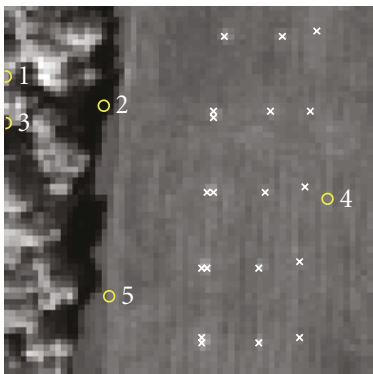

(c)

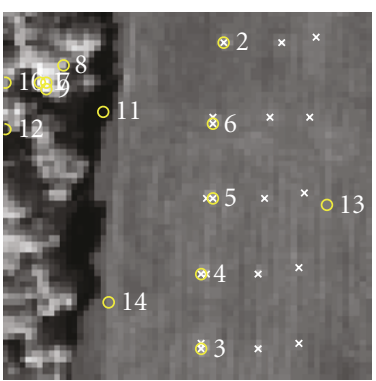

(d)

FIGURE 16: ATGP-generated BKG and target pixels (a) 9 BKG pixels in original data; (b) 9 target pixels in sphered data; (c) 5 BKG pixels not identified as target pixels; (d) 14 pixels obtained by combining the pixels in (b)-(c).

noise bands: bands 1-3 and bands 202-210; and water vapor absorption bands: bands 101-112 and bands 137-153 were removed. So, a total of 169 bands were used in the experiments. The spatial resolution and spectral resolution of this image scene are $1.56 \mathrm{~m}$ and $10 \mathrm{~nm}$, respectively. Within the scene in Figure 15(a) there is a large grass field BKG, and a forest on the left edge. Each element in this matrix is a square panel and denoted by $p_{i j}$ with rows indexed by $i$ and columns indexed by $j=1,2,3$. For each row $i=$ $1,2, \ldots, 5$, there are three panels painted by the same paint but with three different sizes. The sizes of the panels in the first, second, and third columns are $3 \mathrm{~m} \times 3 \mathrm{~m}, 2 \mathrm{~m} \times 2 \mathrm{~m}$, and $1 \mathrm{~m} \times 1 \mathrm{~m}$, respectively. Since the size of the panels in the third column is $1 \mathrm{~m} \times 1 \mathrm{~m}$, they cannot be seen visually from Figure 15(a) due to the fact that its size is less than the $1.56 \mathrm{~m}$ pixel resolution. For each column $j=1,2,3$, the 5 panels have same sizes but in five different paints. However, it should be noted that the panels in rows 2 and 3 were made by the same material with two different paints. Similarly, it is also the case for panels in rows 4 and 5 . Nevertheless, they were still considered as different panels but our experiments will demonstrate that detecting panels in row 5 (row 3 ) may also have effect on detection of panels in row 4 (row 2). The $1.56 \mathrm{~m}$-spatial resolution of the image scene suggests that most of the 15 panels are one pixel in size except that the panels in the 1st column with the 2nd, 3 rd, 4th, 5th rows which are two-pixel panels, denoted by $p_{211}, p_{221}, p_{311}, p_{312}, p_{411}, p_{412}, p_{511}, p_{521}$. As a result, there are a total 19 panel pixels. Figure 15(b) shows the precise spatial locations of these 19 panel pixels where red pixels ( $R$ pixels) are the panel center pixels and the pixels in yellow ( $Y$ pixels) are panel pixels mixed with the BKG. Figure 15(c) shows the spectra of five panel signatures $p_{1}, p_{2}, p_{3}, p_{4}, p_{5}$ obtained by averaging the center $R$ panel pixels for each of five rows.

First of all, the VD estimated for this scene, $n_{\mathrm{VD}}$ was 9 with the false alarm probability $P_{F} \leq 10^{-3}$. Figure 16(a) shows the 9 target pixels which were extracted directly from the original data by the ATGP and can be considered as a set of BKG (BKG) pixels $S^{\mathrm{BKG}}=\left\{\mathbf{b}_{j}^{\mathrm{ATGP}}\right\}_{j=1}^{9}$ and in which three panel pixels from rows 1,3 , and 5 were included. Figure 16(b) shows the 9 target pixels extracted from the sphered data by the ATGP which included five panel pixels extracted from each of five rows and can be considered as a set of target pixels $S^{\text {target }}=\left\{\mathbf{t}_{j}^{\text {ATGP }}\right\}_{j=1}^{9}$. Figure 16(c) singled out the 5 pixels which were identified as BKG pixels $\widetilde{S}^{\text {BKG }}=\left\{\tilde{\mathbf{b}}_{i}^{\text {ATGP }}\right\}$ by removing the 4 target pixels with a similarity measure such as SAM and Figure 16(d) shows a total number of 14 pixels obtained by combining the BKG set $\widetilde{S}^{\mathrm{BKG}}$ in Figure $16(\mathrm{c})$ with the target set $S^{\text {target }}$ in Figure 16(b) into a BKG-target merged set $\widetilde{S}^{\text {BKG }} \cup S^{\text {target }}$ to be used for spectral unmixing where the numbers in the figures indicated the orders of pixels extracted by the ATGP.

Similarly, Figures $17(\mathrm{~d})-18(\mathrm{~d})$ also show the results produced by the UNCLS with 9 target pixels and 5 BKG pixels extracted and by the UFCLS with 9 target pixels and $6 \mathrm{BKG}$ pixels extracted. Since the target pixels of interest are those extracted by the three LS-based algorithms in Figures 16(b), 17(b), and 18(b) from the sphered data they should have included five pure targets pixels that corresponded to all the five pure panel signatures which was exactly the case where these five pure target pixels, $p_{11}, p_{221}, p_{312}, p_{411}$, and $p_{521}$ were found and identical in Figures 16(b), 17(b), and 18(b). Additionally, among these five pure target pixels, $p_{11}, p_{312}$, and $p_{521}$ were the only three target pixels extracted as BKG pixels in the original data in Figures 16(a), 17(a), and 18(a). This was due to the fact that the panel pixels in rows 2 and 4 have very similar signatures to those in rows 3 and 5, respectively, according to the ground truth in which case they were not extracted as endmembers. Interestingly, these three pure target pixels will be the only endmembers extracted by any endmember extraction algorithms except the dimensionality reduction is performed by the independent component analysis (ICA) as shown in the following section.

6.1.1. Endmember Extraction. Once again experiments were also conducted for endmember extraction using the PPI and N-FINDR to extract endmembers from the HYDICE scene in Figure 15(a). Figures 19(a) and 20(a) show the pixels with their PPI counts greater than 0 extracted by PPI with using 500 skewers and 6 endmembers extracted by the N-FINDR where both used MNF to reduce data dimensionality to 9 . As we can see from Figure 20(a) the N-FINDR could only extract two pure panel pixels, $p_{312}$, and $p_{521}$ corresponding 


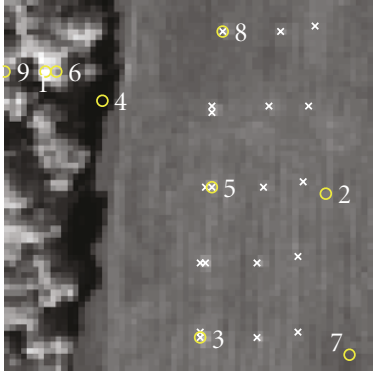

(a)

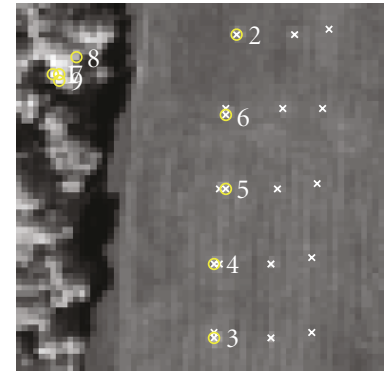

(b)

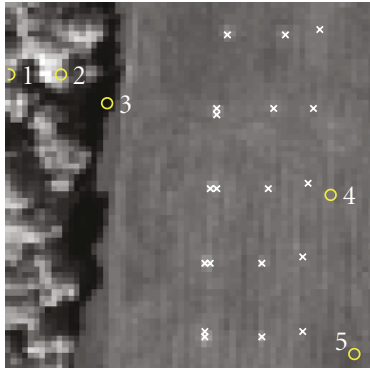

(c)

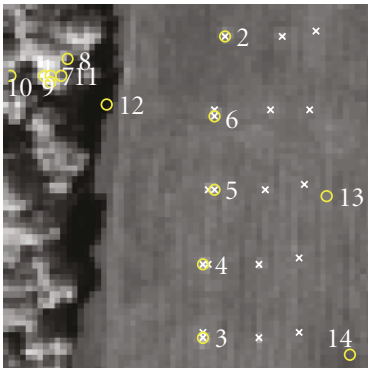

(d)

FIGURE 17: UNCLS-generated BKG and target pixels (a) 9 BKG pixels in original data; (b) 9 target pixels in sphered data; (c) 5 BKG pixels not identified as target pixels; (d) 14 pixels obtained by combining the pixels in (b)-(c).

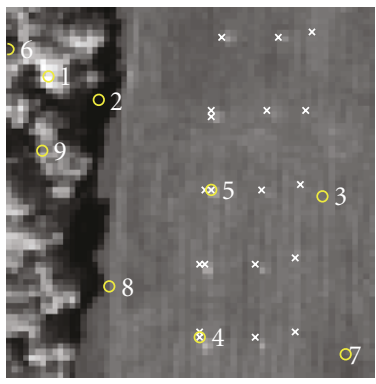

(a)

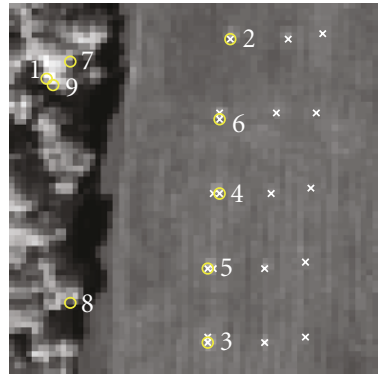

(b)

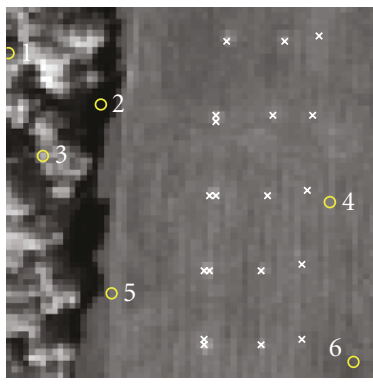

(c)

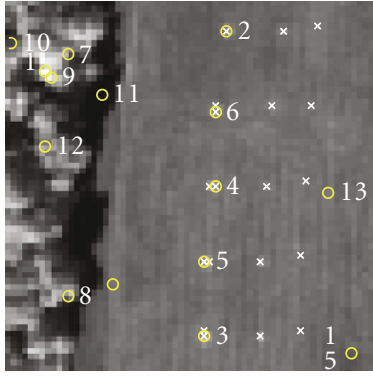

(d)

FIGURE 18: UFCLS-generated BKG and target pixels, (a) 9 BKG pixels in original data; (b) 9 target pixels in sphered data; (c) 6 BKG pixels not identified as target pixels; (d) 15 pixels obtained by combining the pixels in (b)-(c).

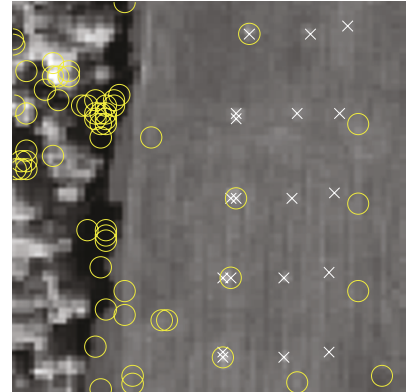

(a) MNF on original data

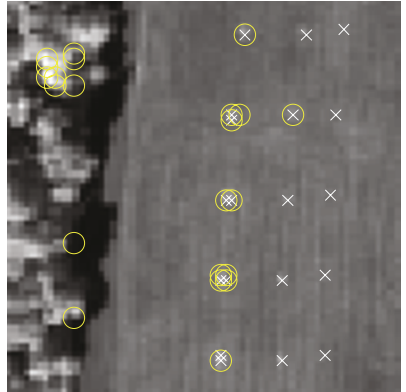

(b) ICA on original data

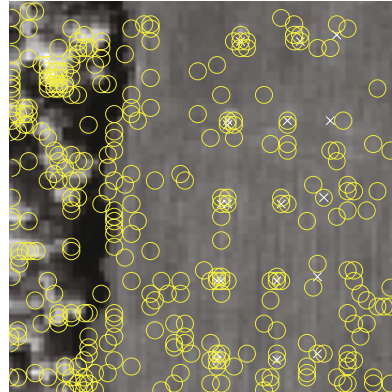

(c) Sphered data

FIGURE 19: Endmember extraction results by PPI using 500 skewers.

to endmembers which are among the $9 \mathrm{BKG}$ pixels extracted in Figures 16(a), 17(a), and 18(a).

However, if we used the ICA instead of MNF to perform dimensionality reduction prior to endmember extraction, the PPI and N-FINDR were able to extract four pure target pixels, $p_{11}, p_{312}, p_{411}$, and $p_{521}$ in Figures $19(\mathrm{~b})$ and $20(\mathrm{~b})$. As a matter of fact, as shown in [20], the PPI and NFINDR would fail to extract all the five endmembers if the dimensionality reduction was performed by 2 nd order statistics transforms such as principal components analysis (PCA), MNF, or singular value decomposition (SVD). With an interesting twist by applying the PPI and N-FINDR to the sphered data without dimensionality reduction as the USTFAs did for Figures 16-18, both were also able to extract all the five endmembers as shown in Figures 19(c) and 20(c). In other words, the only way for an endmember extraction algorithm to succeed in extracting all the five endmembers is either to use ICA for dimensionality reduction or to use the sphered data instead of the original data where in both cases the data characterized by 1st and 2nd order statistics have been removed prior to endmember extraction. This intriguing finding showed that using the ICA as dimensionality reduction has the same effect as applying endmember extraction algorithms such as PPI, N-FINDR, or 


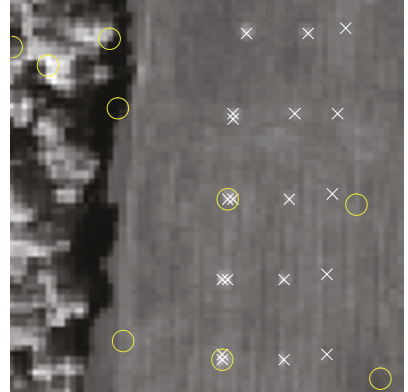

(a) MNF on original data

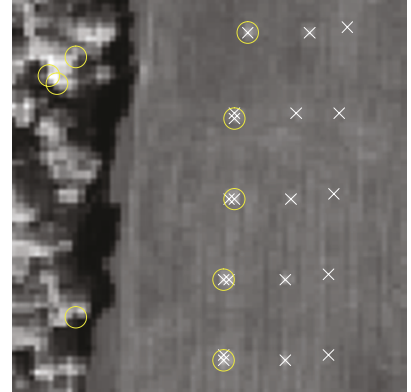

(b) ICA on original data

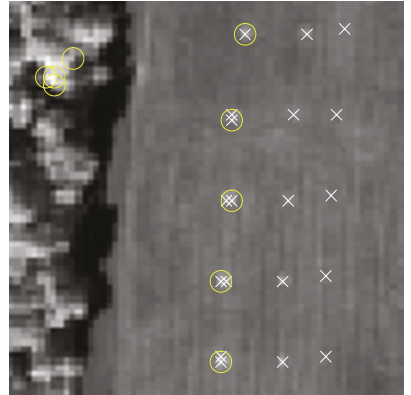

(c) Sphered data

FIgURE 20: 9 endmembers extracted by N-FINDR.
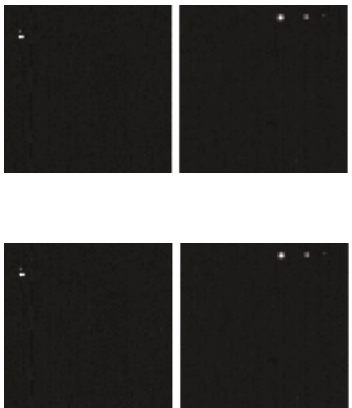
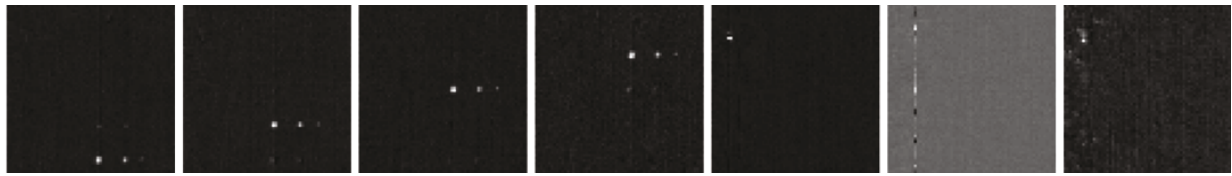

(a) CEM in conjunction with ATGP-USTFA or with UNCLS-USTFA
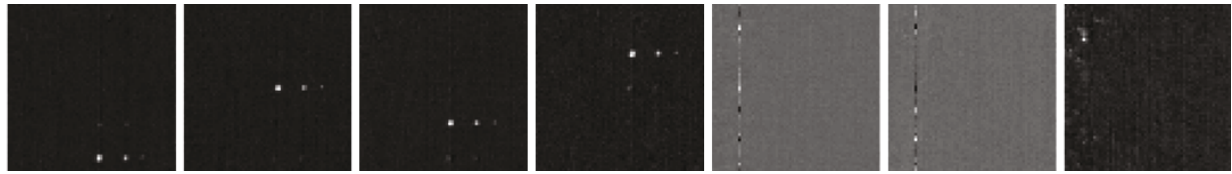

(b) CEM in conjunction with UFCLS-USTFA

Figure 21: CEM detection results using the 9 targets of interest generated by the ATGP, UNCLS, and UFCLS in Figures 16(b), 17(b), and $18(\mathrm{~b})$.

the three USTFAs to the sphered data where both ICA and sphered data retain targets of interest characterized by highorder statistics. As a result, the proposed three USTFAs can be also used for endmember extraction.

6.1.2. Unsupervised Target Detection. This section conducts experiments for unsupervised target detection performed by the CEM using a posteriori target knowledge produced by the three USTFAs from the sphered data. The results are shown in Figures 21(a) and 21(b) where the desired target signatures used by the CEM were the targets of interest extracted in Figures 16(b), 17(b), and 18(b).

In order to further make comparison with anomaly detection, the RXD was implemented and its result is shown in Figure 22.

By visually comparing Figure 22 to Figure 21, the RXD missed all the five subpxiel panels in the 3rd column while the CEM was able to extract all these five subpixel panels using the a posteriori target information provided by the three USTFAs. Most importantly, the RXD could not discriminate the targets it detected. This is not the case for the CEM which used each of a posteriori target knowledge to discriminate among targets found by a USTFA.

6.1.3. Linear Spectral Unmixing. In the unsupervised target detection discussed above the targets of interest were those found by an USTFA from the sphered data so as to achieve

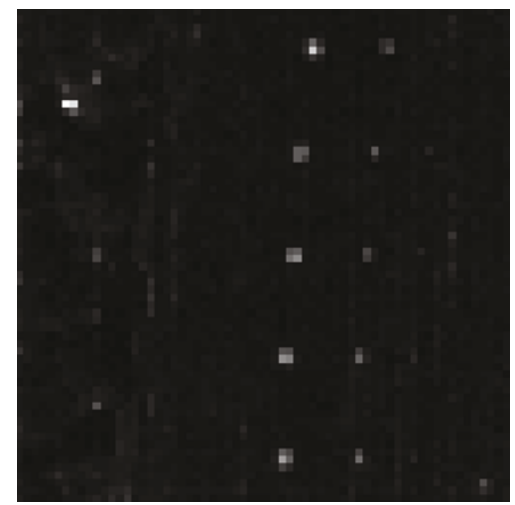

Figure 22: RXD result.

unsupervised target detection rather than target classification. In this section, we further perform target classification by LSU. In order for LSU to perform effectively, the targets signatures used to form the signature matrix $\mathbf{M}$ must include all the targets pixels $\left\{\mathbf{t}_{j}^{\mathrm{LS}}\right\}_{j=1}^{n_{\mathrm{VD}}}$ and BKG pixels $\left\{\tilde{\mathbf{b}}_{i}^{\mathrm{LS}}\right\}_{i=1}^{n_{\mathrm{BKG}}}$ to represent the entire data where the target pixels $\left\{\mathbf{t}_{j}^{\mathrm{LS}}\right\}_{j=1}^{n_{\mathrm{VD}}}$ are the target signatures we would like to classify and the BKG pixels $\left\{\tilde{\mathbf{b}}_{i}^{\mathrm{LS}}\right\}_{i=1}^{n_{\mathrm{BKG}}}$ are considered as undesired signatures which can be removed to enhance target classification performance. The three LSU methods, LSOSP, NCLS, and FCLS were used 

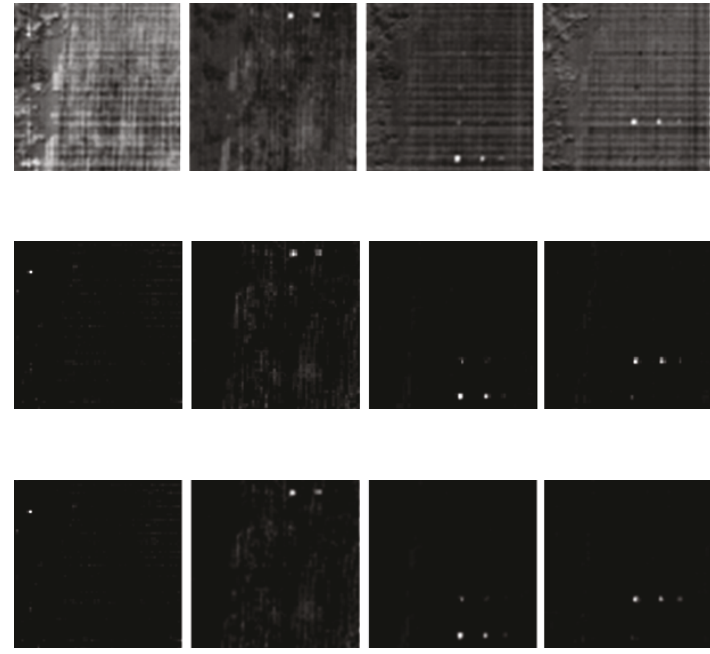
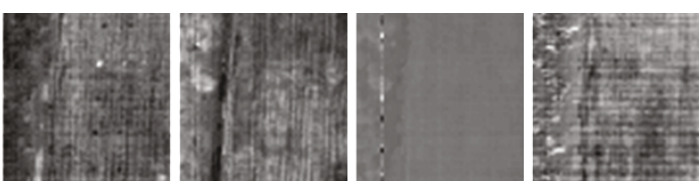

(a) LSOSP
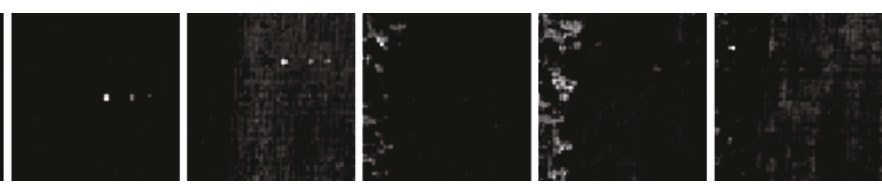

(b) NCLS
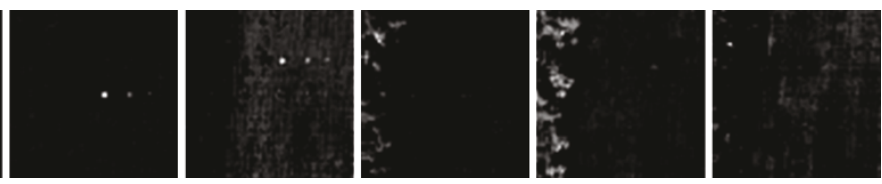

(c) FCLS

FIGURE 23: 9 target classes obtained by LSOSP, NCLS, and FCLS using the target pixels generated by ATGP-USTFA.
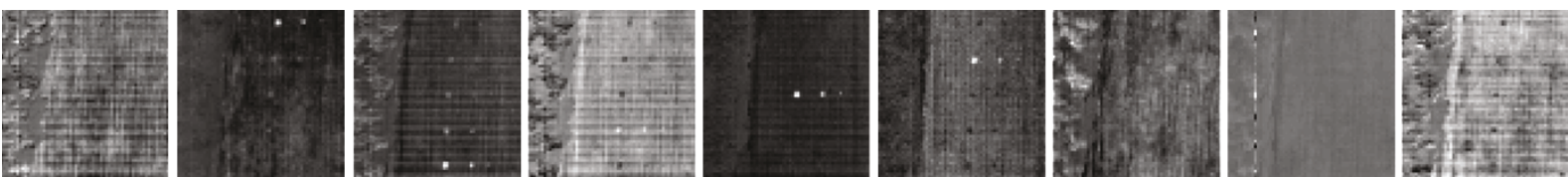

(a) LSOSP
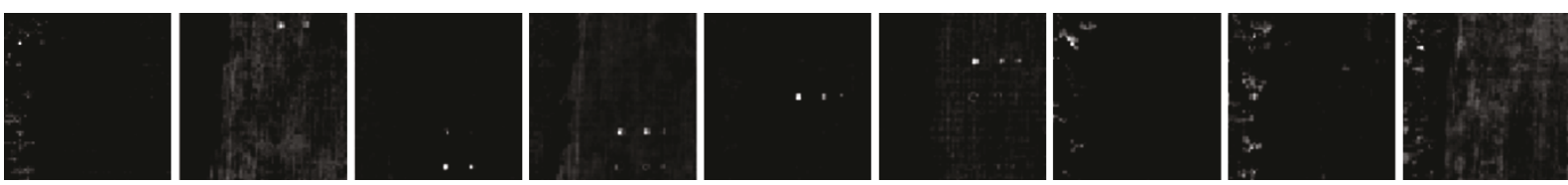

(b) NCLS
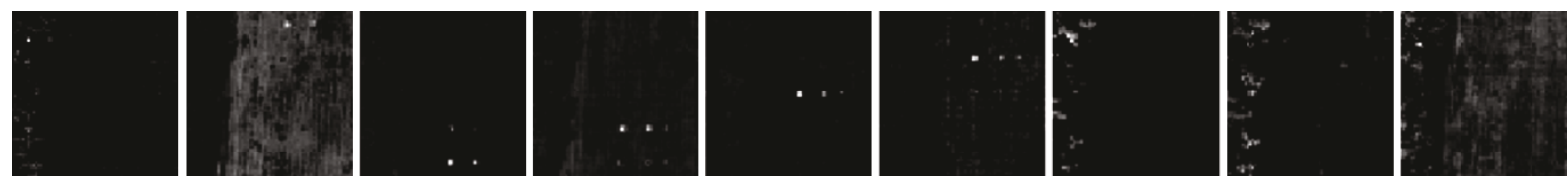

(c) FCLS

FIGURE 24: 9 target classes obtained by LSOSP, NCLS, and FCLS using the target pixels generated by UNCLS-USTFA.

to unmix high-order target pixels $\left\{\mathbf{t}_{j}^{\mathrm{L} S}\right\}_{j=1}^{n_{\mathrm{VD}}}$ extracted from the sphered data, each of which is considered to represent one specific target class. Figures 23(a)-23(c) and 25(a)-25(c) show their corresponding results where figures labeled by (a), (b), and (c) were unmixed results by LSOSP, NCLS, and FCLS, respectively.

Obviously, the results obtained by the NCLS and the FCLS performed better than that by the LSOSP in Figures 23-25 due to the imposed constraints on abundance fractions.

Interestingly, while the results in Figures 23(b) and 23(c) were similar to the results obtained in Figures 24(b)-24(c) and 25(b)-25(c), the umixed results obtained by the LSOSP in Figure 25(a) were slightly better than those in Figures 23(a) and 24(a) in terms of detection of 15 panels in five rows. This improvement was mainly due to the fact that the UFCLS produced $6 \mathrm{BKG}$ pixels rather than $5 \mathrm{BKG}$ pixels produced by the ATGP and UNCLS to perform better BKG suppression.

Since the evaluation of the above unmixed results was performed qualitatively by visual assessment, the conclusions may not be objective. Table 3 further tabulates their quantitative results where the abundance fractions of the 19 panel pixels estimated by the NCLS and FCLS were very close and both the NCLS and the FCLS outperformed the unconstrained LSOSP, even though the NCLS is only a partially abundance constrained method.

Finally, in order to demonstrate that the performance of unsupervised linear hyperspectral unmixing using the designed USTFA is superior to that of supervised linear 

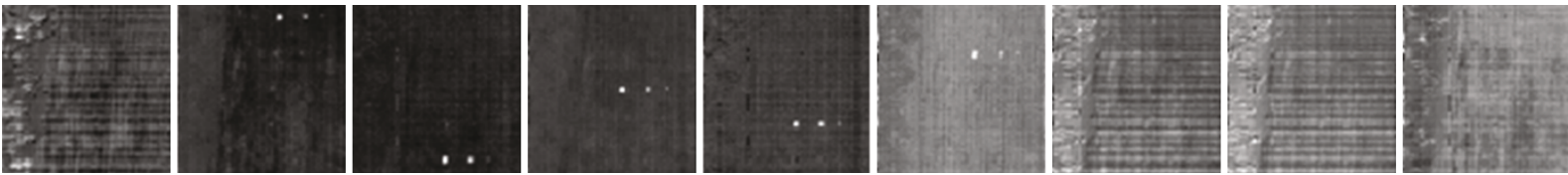

(a) LSOSP
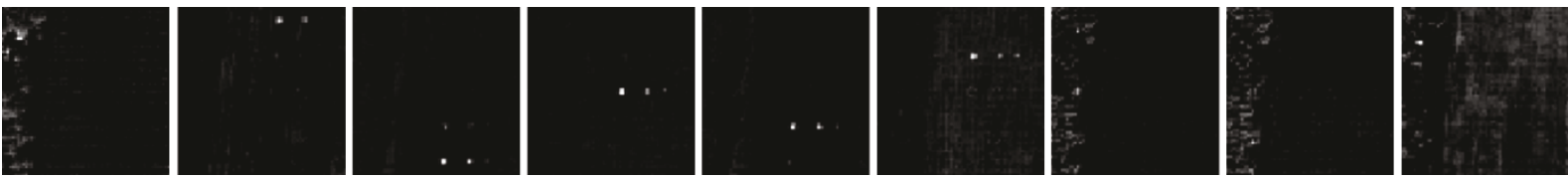

(b) NCLS
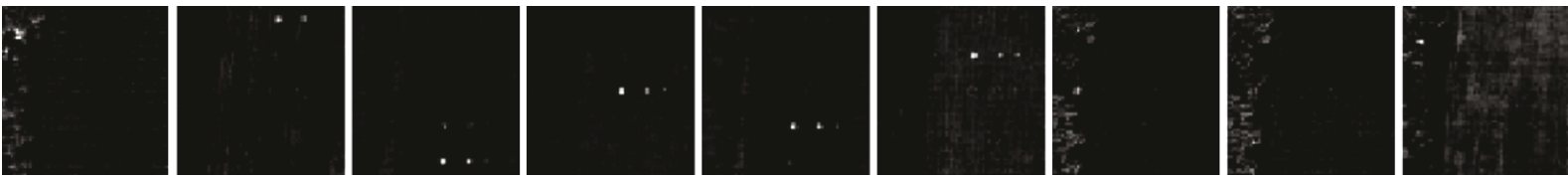

(c) FCLS

FIGURE 25: 9 target classes obtained by LSOSP, NCLS, and FCLS using the target pixels generated by UFCLS-USTFA.

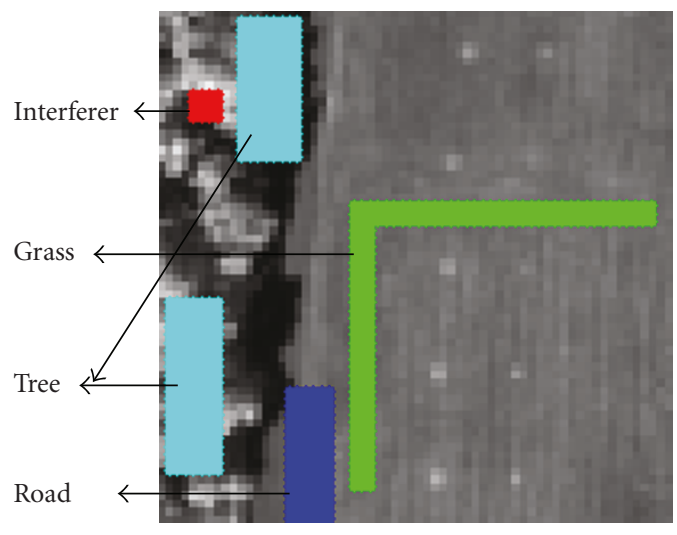

FIGURE 26: Four BKG classes obtained by marked areas.

spectral unmixing, we further conducted experiments for supervised linear spectral unmxing where the five panel signatures were obtained from Figures 15(b) and 15(c) and the other four BKG signatures were obtained by prior knowledge as the areas marked in Figure 26 as interferer, tree, grass, road to make up 5 target classes representing five panel signatures and $4 \mathrm{BKG}$ signatures. These signatures represent exactly 9 signatures estimated by the VD. This fact provides further evidence that VD is an effective estimation method to estimate the number of image endmembers for spectral unmixing.

Figures 27(a)-27(c) show the unmixed results of 19 panel pixels by the three unmixing methods, LSOSP, NCLS, and FCLS where the unmixed results for 4 BKG classes were not displayed because the BKG classes were not of major interest. Also, it should be noted that there are more BKG pixels that can be used for this purpose. As a matter of fact, in [7] there were 34 pixels found by the unsupervised FCLS for spectral unmixing. The results were similar to those presented in our experiments using only 9 image endmembers. In this case,
"9" is probably sufficiently enough for spectral unmixing to perform well.

If we compare the results in Figure 27 to Figures 23-25, it is obvious that the ULSU performed significantly better than its supervised counterparts. Table 4 also tabulates the quantitative results obtained in Figures 27(a)-27(c) in comparison with the results in Table 3 where it clearly showed that the abundance fractions estimated by unsupervised linear unmixing in Table 3 were much more accurate than those obtained by its supervised counterpart in Table 4 .

An interesting finding from Table 4 is that the abundance fractions of panel pixels $p_{412}$ estimated by the NCLS and $p_{411}, p_{412}$ estimated by the FCLS were zero. This was caused by the fact that the five panel signatures $p_{1}, p_{2}, p_{3}, p_{4}, p_{5}$ in Figure 15(c) used for spectral unmixing were not really pure signatures because the panel pixels in the 3rd column that were included for averaging were actually subpixels and not pure signatures. If we conducted the same experiments by using the panel signatures $p_{1}, p_{2}, p_{3}, p_{4}, p_{5}$ that were obtained by averaging only $R$ panel center pixels in the 1 st and 2 nd columns for spectral unmixing, Table 5 tabulates the abundance fractions of 19 panel pixels by the LSOSP, NCLS, and FCLS where the abundance fractions of panel pixels $p_{411}$ and $p_{412}$ were corrected and no longer zero.

A comment on Table 5 is noteworthy. According to the ground truth $p_{411}$ and $p_{412}$ are the panel center pixels. However, from our extensive experience with the HYDICE scene, they are in fact not as pure pixels of $100 \%$ abundance purity as we expect. As a result, even though the NCLS is partially abundance-constrained, it was very comparable to the fully abundance-constrained FCLS in terms of abundance estimation. Nevertheless, both performed significantly better than the abundance-unconstrained LSOSP.

Comparing the results in Table 5 against those in Table 4, it apparently shows that using contaminated or inaccurate prior knowledge may result in significant distortion in quantification of abundance fractions. If we further compare 
TABLE 3: Abundance fractions of 19 panel pixels estimated by LSOSP, NCLS, and FCLS using BKG and target pixels found in Figures 23-25 by ATGP-USTFA, UNCLS-USTFA, and UFCLS-USTFA.

\begin{tabular}{|c|c|c|c|c|c|c|c|c|c|}
\hline & \multicolumn{3}{|c|}{$\tilde{\mathbf{b}}_{i}^{\mathrm{ATGP}}$}$\cup\left\{\mathbf{t}_{j}^{\mathrm{ATGP}}\right\}_{j=1}^{9}$ & \multicolumn{3}{|c|}{$\tilde{\mathbf{b}}_{i}^{\mathrm{UNCLS}}$}$\cup\left\{\mathbf{t}_{j}^{\mathrm{UNCLS}}\right\}_{j=1}^{9}$ & \multicolumn{3}{|c|}{$\tilde{\mathbf{b}}_{i}^{\mathrm{UFCLS}}$}$\cup\left\{\mathbf{t}_{j}^{\mathrm{UFCLS}}\right\}_{j=1}^{9}$ \\
\hline & LSOSP & NCLS & FCLS & LSOSP & NCLS & FCLS & LSOSP & NCLS & FCLS \\
\hline$p_{11}$ & 1.0000 & 1.0000 & 1.0000 & 1.0000 & 1.0000 & 1.0000 & 1.0000 & 1.0000 & 1.0000 \\
\hline$p_{12}$ & 0.4096 & 0.4332 & 0.4120 & 0.3562 & 0.4165 & 0.4001 & 0.4085 & 0.4148 & 0.3850 \\
\hline$p_{13}$ & 0.0002 & 0.0887 & 0.0841 & -0.1073 & 0.0308 & 0.0465 & 0.0142 & 0.0307 & 0.0250 \\
\hline$p_{211}$ & 0.8421 & 0.8403 & 0.8404 & 0.9180 & 0.8413 & 0.8209 & 0.8648 & 0.8384 & 0.8453 \\
\hline$p_{221}$ & 1.0000 & 1.0000 & 1.0000 & 1.0000 & 1.0000 & 1.0000 & 1.0000 & 1.0000 & 1.0000 \\
\hline$p_{22}$ & 0.6164 & 0.6257 & 0.7308 & 0.6351 & 0.6607 & 0.7127 & 0.6990 & 0.6126 & 0.7405 \\
\hline$p_{23}$ & 0.5525 & 0.4774 & 0.4724 & 0.3478 & 0.4168 & 0.4153 & 0.3798 & 0.4471 & 0.4498 \\
\hline$p_{311}$ & 0.8741 & 0.8674 & 0.8627 & 0.9094 & 0.8674 & 0.8628 & 0.8969 & 0.8671 & 0.8634 \\
\hline$p_{312}$ & 1.0000 & 1.0000 & 1.0000 & 1.0000 & 1.0000 & 1.0000 & 1.0000 & 1.0000 & 1.0000 \\
\hline$p_{32}$ & 0.5027 & 0.4249 & 0.4192 & 0.5906 & 0.4713 & 0.4727 & 0.5925 & 0.5149 & 0.4922 \\
\hline$p_{33}$ & 0.2516 & 0.2614 & 0.2655 & 0.3541 & 0.2959 & 0.2929 & 0.3388 & 0.2880 & 0.2886 \\
\hline$p_{411}$ & 1.0000 & 1.0000 & 1.0000 & 1.0000 & 1.0000 & 1.0000 & 1.0000 & 1.0000 & 1.0000 \\
\hline$p_{412}$ & 0.7685 & 0.3137 & 0.3876 & 0.5827 & 0.3222 & 0.3605 & 0.7976 & 0.3407 & 0.3923 \\
\hline$p_{42}$ & 0.8085 & 0.6761 & 0.6657 & 0.7965 & 0.7495 & 0.7485 & 0.8414 & 0.7480 & 0.7477 \\
\hline$p_{43}$ & 0.2363 & 0.1789 & 0.1473 & 0.5047 & 0.2851 & 0.2633 & 0.2790 & 0.1227 & 0.1542 \\
\hline$p_{511}$ & 0.7204 & 0.7224 & 0.7215 & 0.6954 & 0.7245 & 0.7198 & 0.6973 & 0.7213 & 0.7235 \\
\hline$p_{521}$ & 1.0000 & 1.0000 & 1.0000 & 1.0000 & 1.0000 & 1.0000 & 1.0000 & 1.0000 & 1.0000 \\
\hline$p_{52}$ & 0.7645 & 0.7770 & 0.7689 & 0.7027 & 0.7460 & 0.7244 & 0.7228 & 0.7753 & 0.7740 \\
\hline$p_{53}$ & 0.1452 & 0.1545 & 0.1537 & -0.0144 & 0.0000 & 0.0017 & 0.1215 & 0.1471 & 0.1554 \\
\hline
\end{tabular}
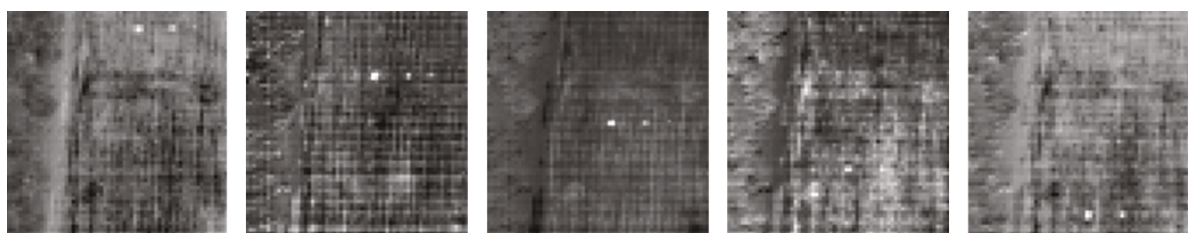

(a) LSOSP
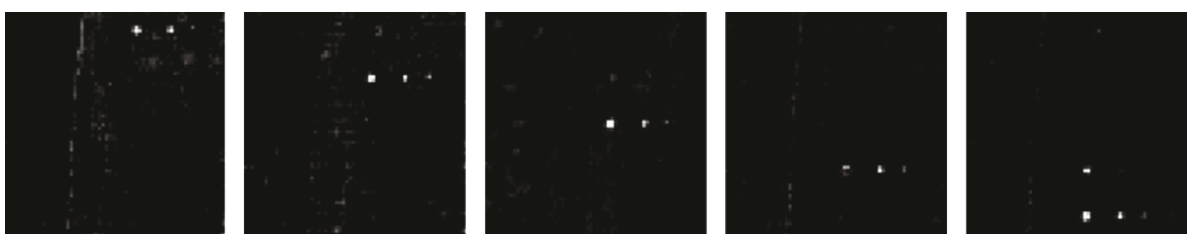

(b) NCLS

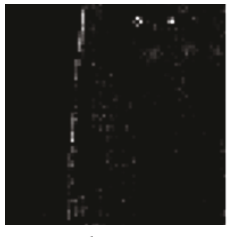

Panels in row 1

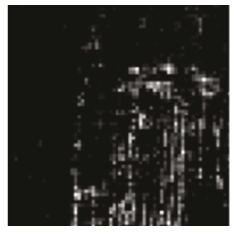

Panels in row 2

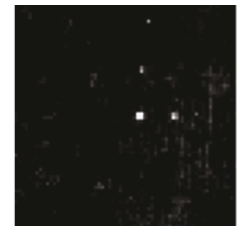

Panels in row 3

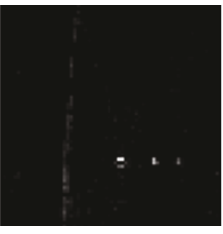

Panels in row 4

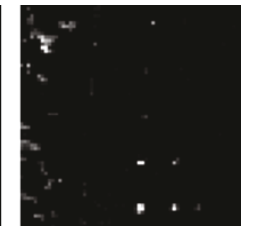

Panels in row 5

(c) FCLS

FIGURE 27: Unmixed results of 15 panels with 19 panel pixels by supervised LSOSP, NCLS, and FCLS using five panel signatures in Figure 17(c) and 4 BKG signatures obtained by marked areas in Figure 24.

the results in Table 3 against those in Tables 4 and 5, the USLU outperformed SLSU significantly. These experiments further demonstrate two facts. One is that SLSU is effective only if the prior knowledge is accurate such as the synthetic image experiments conducted for scenarios TI and TE in
Section 5. Unfortunately, this may not be true when it comes to real world applications where true target knowledge is generally difficult to obtain, if not impossible. Even in the case that prior target knowledge is available, it may not be reliable due to many unknown signal sources that may 
TABLE 4: Abundance fractions of 19 panel pixels in Figures 27(a)27(c) estimated by LSOSP, NCLS, and FCLS.

\begin{tabular}{lccc}
\hline & LSOSP & NCLS & FCLS \\
\hline$p_{11}$ & 1.4475 & 0.8309 & 0.0146 \\
$p_{12}$ & 0.9155 & 0.8510 & 0.8199 \\
$p_{13}$ & 0.6370 & 0.2735 & 0.1946 \\
$p_{211}$ & 1.2384 & 0.8115 & 0.5457 \\
$p_{221}$ & 1.3146 & 0.7945 & 0.3429 \\
$p_{22}$ & 0.8558 & 0.8558 & 0.8524 \\
$p_{23}$ & 0.5912 & 0.4843 & 0.4985 \\
$p_{311}$ & 1.2482 & 0.8809 & 0.8298 \\
$p_{312}$ & 1.4713 & 0.8953 & 0.7912 \\
$p_{32}$ & 0.8240 & 0.5935 & 0.7389 \\
$p_{33}$ & 0.4565 & 0.2761 & 0.2705 \\
$p_{411}$ & 1.2356 & 0.1617 & 0.0000 \\
$p_{412}$ & 1.1672 & 0.0000 & 0.0000 \\
$p_{42}$ & 1.2331 & 0.9555 & 0.4772 \\
$p_{43}$ & 0.3641 & 0.2393 & 0.2003 \\
$p_{511}$ & 1.1770 & 0.9599 & 0.9759 \\
$p_{521}$ & 1.4698 & 0.9551 & 1.0000 \\
$p_{52}$ & 1.0760 & 0.9925 & 1.0000 \\
$p_{53}$ & 0.2772 & 0.2029 & 0.1763 \\
\hline
\end{tabular}

TABLE 5: Abundance fractions of 19 panel pixels estimated by LSOSP, NCLS, and FCLS using panel signatures in Figure 15(c).

\begin{tabular}{lccc}
\hline & LSOSP & NCLS & FCLS \\
\hline$p_{11}$ & 1.2635 & 0.9551 & 0.7606 \\
$p_{12}$ & 0.7366 & 0.6226 & 0.5376 \\
$p_{13}$ & 0.4053 & 0.1302 & 0.0079 \\
$p_{211}$ & 1.0853 & 0.9929 & 0.9085 \\
$p_{221}$ & 1.1652 & 0.9530 & 0.7869 \\
$p_{22}$ & 0.7495 & 0.8130 & 0.8240 \\
$p_{23}$ & 0.4578 & 0.4018 & 0.4231 \\
$p_{311}$ & 1.0584 & 0.9139 & 0.9136 \\
$p_{312}$ & 1.2402 & 0.9292 & 0.9024 \\
$p_{32}$ & 0.7014 & 0.4699 & 0.4482 \\
$p_{33}$ & 0.3756 & 0.2072 & 0.2100 \\
$p_{411}$ & 1.0532 & 0.9104 & 0.5087 \\
$p_{412}$ & 0.9507 & 0.4329 & 0.4384 \\
$p_{42}$ & 0.9962 & 0.7863 & 0.7575 \\
$p_{43}$ & 0.2684 & 0.1896 & 0.1571 \\
$p_{511}$ & 0.9531 & 0.8304 & 0.8304 \\
$p_{521}$ & 1.1738 & 1.0295 & 1.0000 \\
$p_{52}$ & 0.8732 & 0.9354 & 0.9353 \\
$p_{53}$ & 0.2303 & 0.1596 & 0.1372 \\
\hline
\end{tabular}

contaminate the knowledge. This leads to the second fact that to avoid using unreliable prior knowledge ULSU certainly provides a better alternative to SLSU.

6.2. AVIRIS Data. The second data is an Airborne Visible InfraRed Imaging Spectrometer (AVIRIS) image scene shown in Figure 28(a) which is the Lunar Crater Volcanic
TABLE 6: SAM values of six signatures in Figure 28(b).

\begin{tabular}{lcccccc}
\hline & Cinder & Playa & Rhyolite & Shade & Vegetation & Anomaly \\
\hline Cinder & 0 & 0.292 & 0.207 & 0.273 & 0.221 & 0.397 \\
Playa & 0.292 & 0 & 0.111 & 0.141 & 0.114 & 0.119 \\
Rhyolite & 0.207 & 0.111 & 0 & 0.166 & $\mathbf{0 . 0 4 1}$ & 0.227 \\
Shade & 0.273 & 0.141 & 0.166 & 0 & 0.148 & 0.189 \\
Vegetation & 0.221 & 0.114 & $\mathbf{0 . 0 4 1}$ & 0.148 & 0 & 0.224 \\
Anomaly & 0.397 & 0.119 & 0.227 & 0.189 & 0.224 & 0 \\
\hline
\end{tabular}

Field (LCVF) located in Northern Nye County, NV. Atmospheric water bands and low SNR bands have been removed from the data, reducing the image cube from 224 to 158 bands. The image in Figure 28 has $10 \mathrm{~nm}$ spectral resolution and $20 \mathrm{~m}$ spatial resolution. The ground truth of this image scene identifies five areas of interest red oxidized basaltic cinders, rhyolite, playa (dry lake), vegetation, and shade and their marked spectral signature are shown in Figure 28(b) for data analysis. It should be noted that there is a two-pixel size anomaly which cannot be identified by visual inspection.

The VD estimated for this scene using the HFC method is 4 for $P_{F} \leq 10^{-3}$. Since the ground truth of this image scene is limited, only the application of LSU was conducted for experiments. Figures 29(a) and 29(b) show 4 pixels extracted by ATGP each from the original and sphered image data where the sample labeled by 1 extracted in Figure 29(a) was also extracted as the sample labeled by 1 in Figure 29(b). Interestingly, among the 4 extracted samples in Figure 29(b), the samples labeled by 1 and 2 were identified by the SAM to belong to the same target class which actually comprises of one single target, a two-pixel anomaly. These two pixels are the only samples that were identified as target pixels. Therefore, a total of 7 samples ( 3 for BKG pixels and 4 for target pixels), each of which represents a spectral class, were used for SLSU. According to the ground truth, samples $1-2,3,6,7$ represent anomalies, vegetation, cinder, playa, and shade, respectively. Figure 30 shows the results unmixed by the LSOSP, NCLS, and FCLS where FCLS seemed to perform the best where the two anomalous target pixels which are supposed to belong to the same class were also unmixed in two different classes. As expected, two unmixed anomaly pixels in Figure 30 were very similar. Interestingly, according to the results in Figure 30, the vegetation and rhyolite were unmixed into the same class due to the fact that these two spectral signature shapes are very similar and close according to their normalized spectra in Figure 28(c). Table 6 further tabulates the SAM values of six signatures in Figure 28(b) where the vegetation and rhyolite spectral signatures are indeed very close. Such subtle difference can be only seen from the prior knowledge provided in Figure 28(c).

Similarly, UNCLS-USTFA generated 4 BKG pixels in Figure $31(\mathrm{a})$ and 4 target pixels in Figure 31(b) where 2 pixels were in Figure 31(c) were identified as BKG pixels. By combining pixels in Figures 31(b) and 31(c) a total of 6 pixels were used by LSU as signatures for unmixing where pixels 1 and 2 are anomalies, pixels 3, 5, 6 represent signatures of 

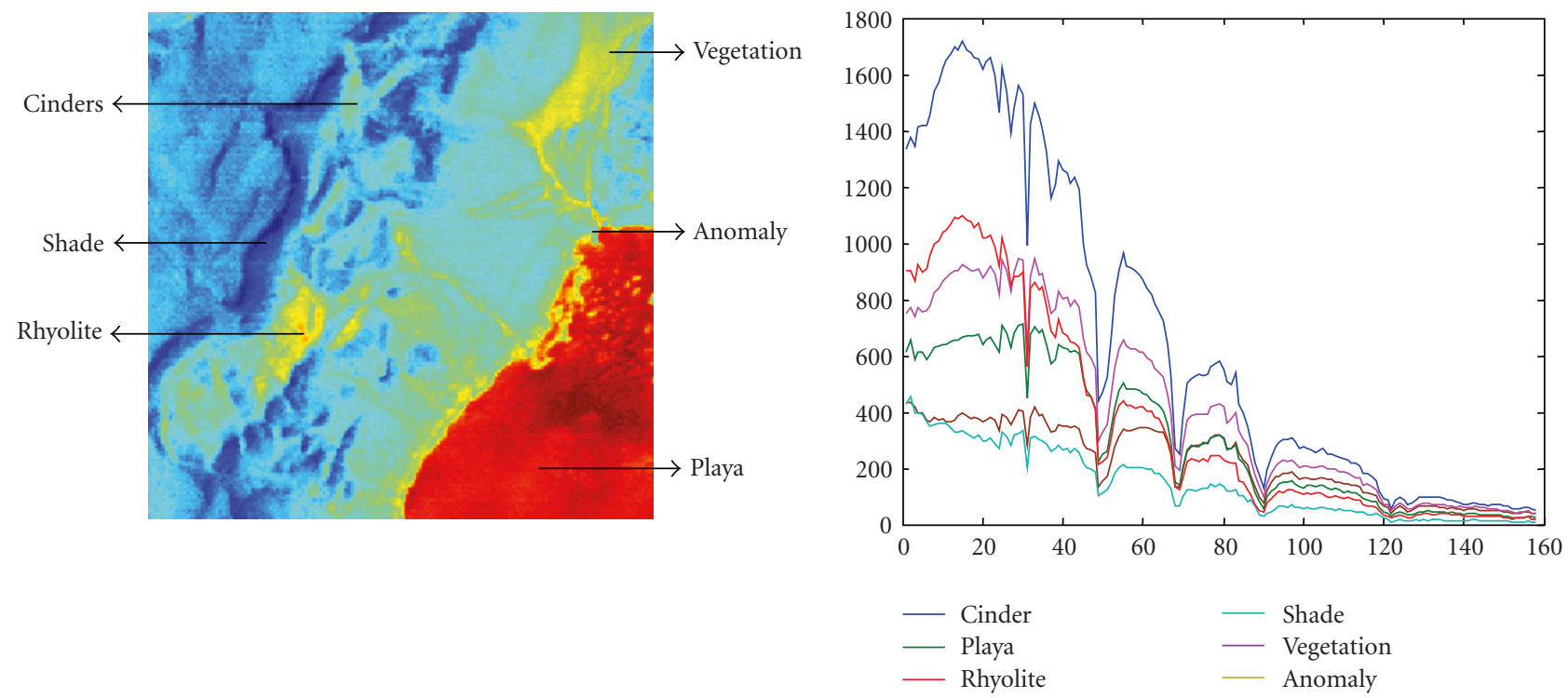

(a)

(b) Original spectra

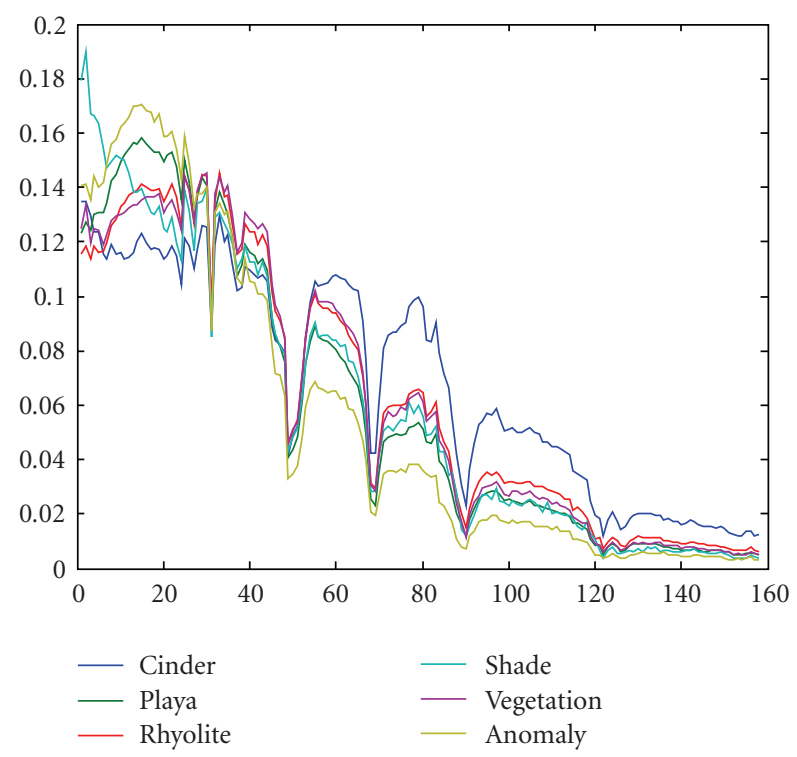

(c) Normalized spectra

FIgURE 28: AVARIS LCVF scene.

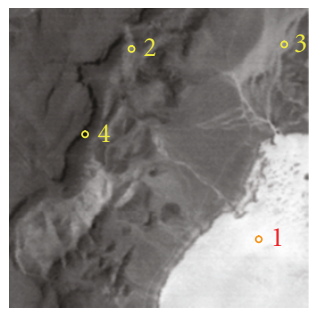

(a) 4 BKG pixels

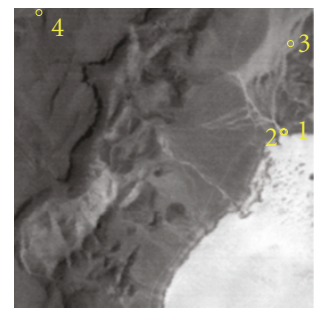

(b) 4 target pixels

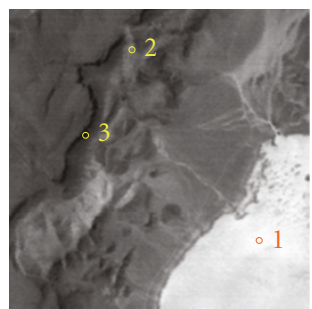

(c) 3 BKG pixels

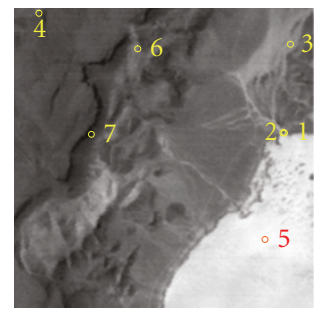

(d) 7 pixels used for unmixing

Figure 29: ATGP-USTFA generated BKG and target pixels (a) 4 BKG pixels in original data; (b) 4 target pixels in sphered data; (c) 1 BKG pixel not identified as target pixels; (d) 7 pixels obtained by combining the pixels in (b)-(c). 

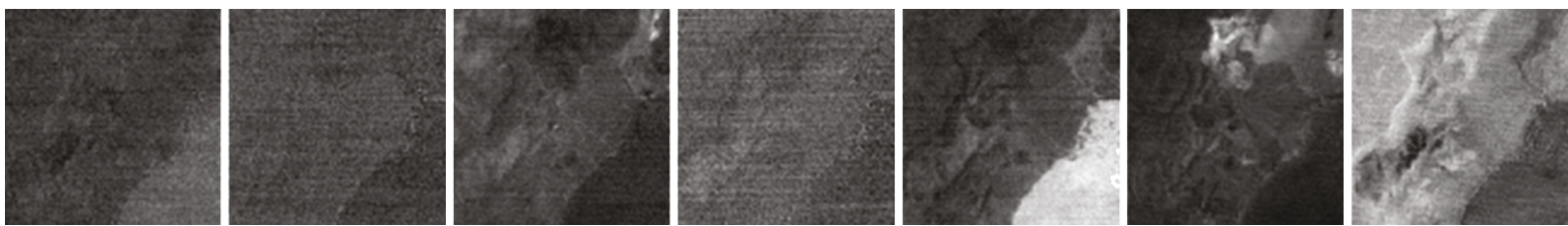

(a) LSOSP
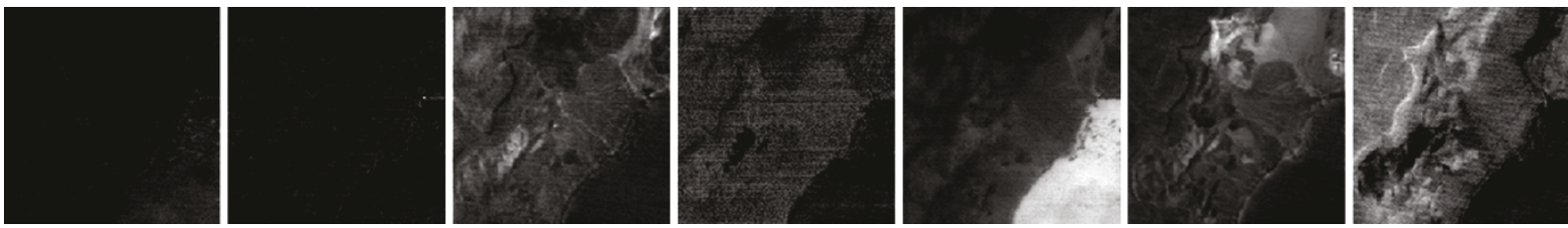

(b) NCLS

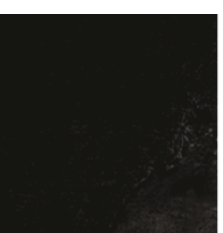

Anomaly

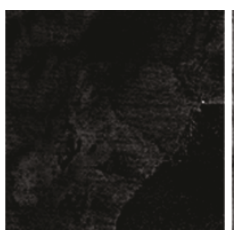

Anomaly

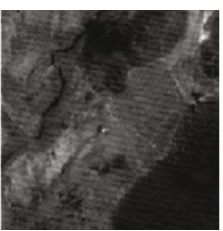

Vegetation/rhyolite

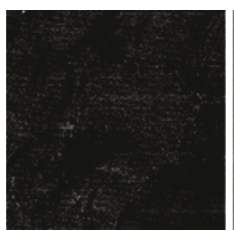

(c) FCLS

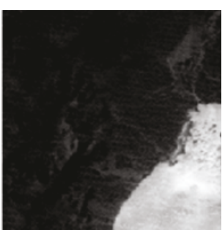

Playa

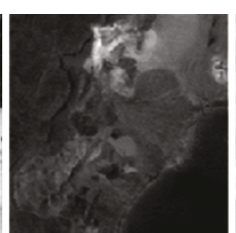

Cinder

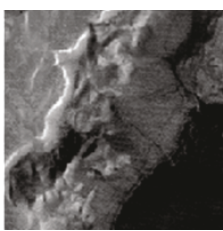

Shade

Figure 30: LSOSP, NCLS, and FCLS unmixed results using the samples extracted in Figure 29(d).

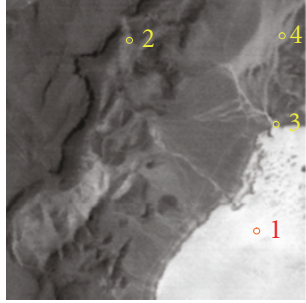

(a) 4 BKG pixels

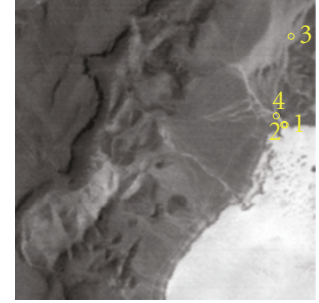

(b) 4 target pixels

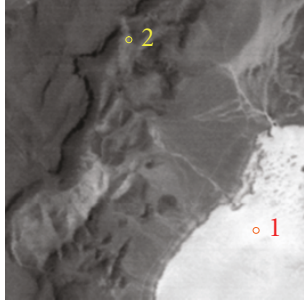

(c) 2 BKG pixels

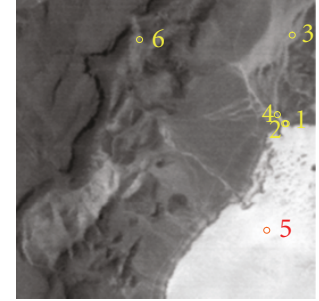

(d) 6 pixels used for unmixing

FIGURE 31: UNCLS-generated BKG and target pixels (a) 4 BKG pixels in original data; (b) 4 target pixels in sphered data; (c) 2 pixels identified as BKG pixels; (d) a total of 6 pixels obtained by combining the pixels in (b)-(c).

vegetation, playa and cinders, respectively. Figure 32 shows the unmixed results of each of the 6 signatures by LSOSP, NCLS, and FCLS where once again FCLS was the best and the vegetation and rhyolite were also unmixed into the same class.

Following the treatment of ATGP-USTFA and UNCLSUSTFA Figure 33 shows UFCLS-USTFA-generated 4 BKG pixels in Figure 33(a) and 4 target pixels in Figure 33(b) where 3 pixels in Figure 33(c) were identified as BKG pixels. By combining pixels in Figures 33(b) and 33(c) a total of 7 pixels were used by LSU as signatures for unmixing where pixels 1(4), 3, 5, 7 represent anomalies and signatures of vegetation, playa, and cinders, respectively. Figure 34 shows the unmixed results of each of the 6 signatures by LSOSP, NCLS, and FCLS where once again FCLS was the best and the vegetation and rhyolite were also unmixed into the same class. Interestingly, without prior knowledge the USTFA using ATGP, UNCLS, and UFCLS missed the signature of rhyolite in Figures 29, 31, and 33, but it did pick up two anomalous pixels which cannot be identified by visual inspection. The reason of missing rhyolite is because its spectral signature shape is very similar to that of vegetation according to the six spectral signatures in Figure 28(c) and their SAM values in Table 6. In this case, extracting vegetation also extracts the rhyolite, a fact demonstrated in Figures 30, 32, and 34. This experiment demonstrates that spectral signature shapes are more crucial than spectral signature amplitudes.

In order to see effectiveness of using USTFA-generated pixels as target pixels to perform ULSU, supervised LSOSP, NCLS, and FCLS using the five signatures, cinders, playa, rhyolite, shade, and vegetation in Figure 28(b) were performed for SLSU for comparison and Figure 35 shows their unmixed results. 

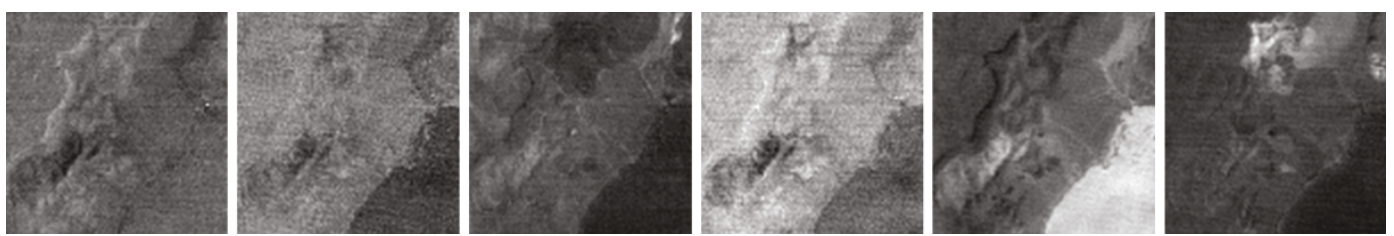

(a) LSOSP
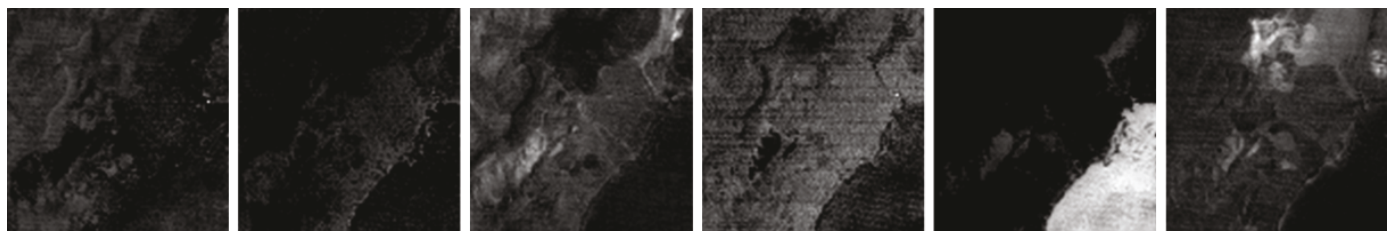

(b) NCLS

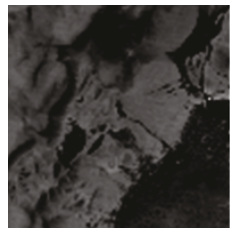

Anomaly

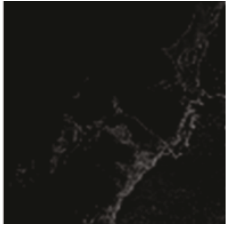

Anomaly

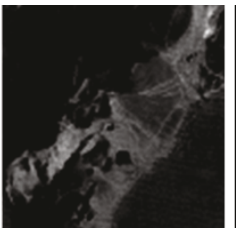

Vegetation/rhyolite

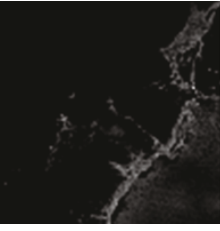

c) FCLS

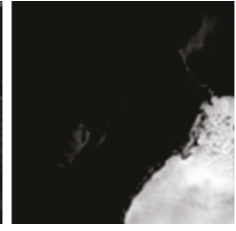

Playa

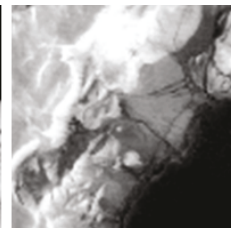

Cinder

FIGURE 32: LSOSP, NCLS, and FCLS unmixed results using signatures of samples in Figure 31(d).

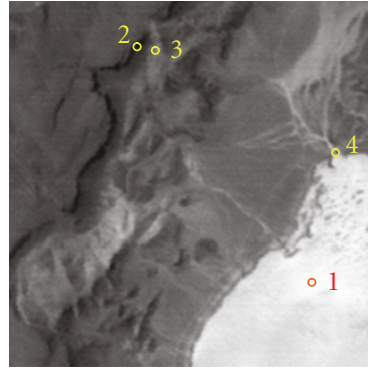

(a) 4 BKG pixels

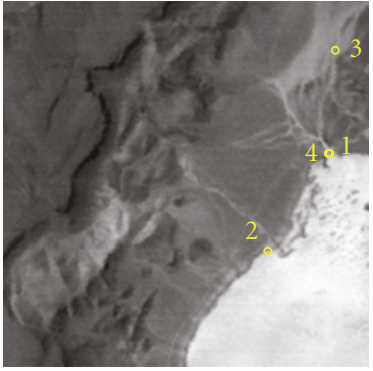

(b) 4 target pixels

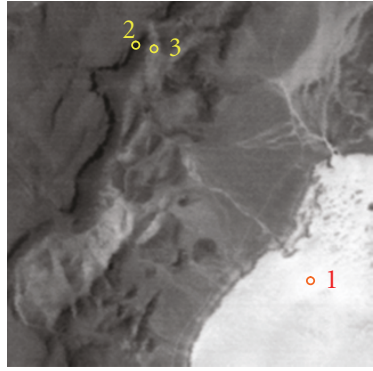

(c) 3 BKG pixels

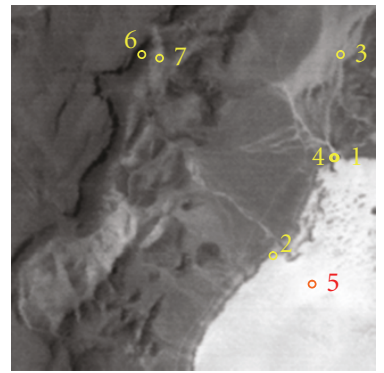

(d) 7 pixels used for unmixing

FIGURE 33: UFCLS-generated BKG and target pixels (a) 4 BKG pixels in original data; (b) 4 target pixels in sphered data; (c) 3 BKG pixels not identified as target pixels; (d) 7 pixels obtained by combining the pixels in (b)-(c).

By comparing the unmixed results in Figures 30, 32, and 34 to that in Figure 35 three interesting findings are worth being mentioned.

(1) The unmixed results of cinders, playa, shade produced by USTFA-based ULSU were much better than that by SLSU using the prior knowledge in Figure 28(b).

(2) Due to unavailability of prior knowledge, there is no way for an unsupervised algorithm to differentiate rhyolite from vegetation because their spectral signatures are so similar according to Table 6 that the USTFA considered both signatures belonging to the same spectral class. As a result, these two signatures were unmixed in the same class as demonstrated in
Figures 30, 32, and 34. However, with using the prior knowledge provided in Figure 28(b), Figure 35 was able to distinguish the rhyolite from the vegetation.

(3) On the other hand, since the two-pixel size anomaly is not visible by inspection or prior knowledge, this target was not shown in Figure 35. But its significance was extracted by all the three unsupervised algorithms, ATGP, UNCLS, and UFCLS. As a matter of fact, this target was picked up as the first target pixels from sphered data. This indicated that this unknown target was crucial and critical for unsupervised hyperspectral target analysis. Unfortunately, it was missed in supervised target analysis in Figure 35 since it cannot be known by visual assessment. 

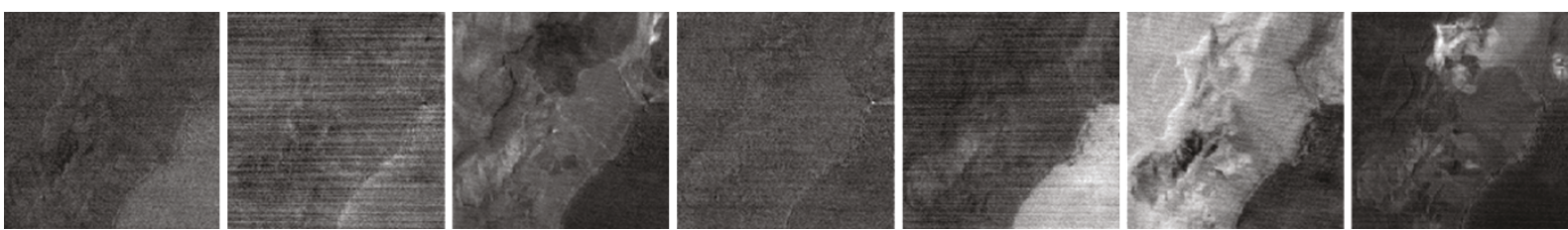

(a) LSOSP
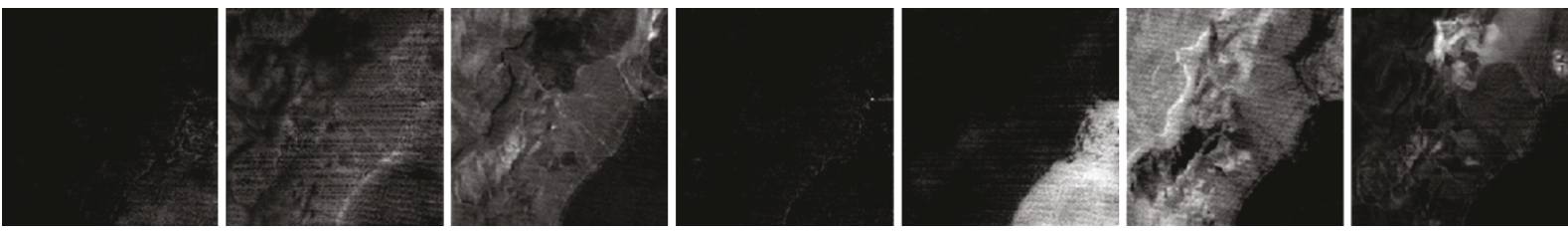

(b) NCLS

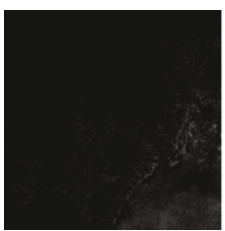

Anomaly
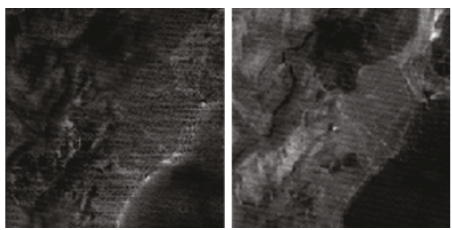

Vegetation/rhyolite

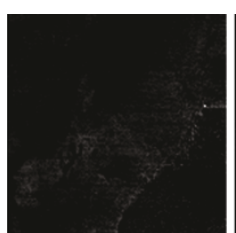

Anomaly

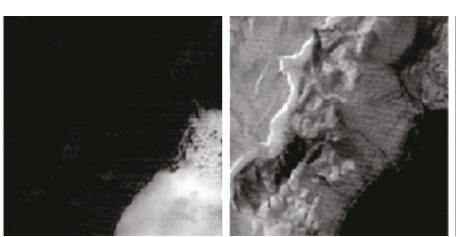

Shade

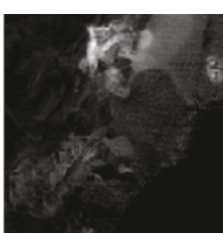

Cinders

(c) FCLS

Figure 34: LSOSP, NCLS, and FCLS unmixed results using signatures of samples in Figure 33(d).
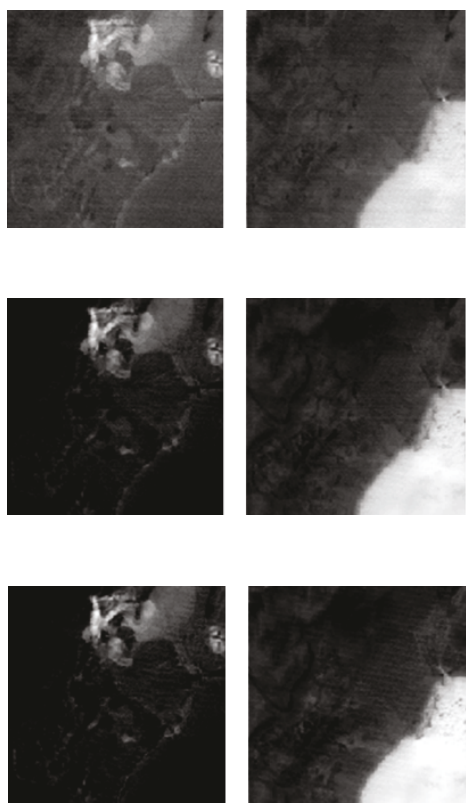

Cinders

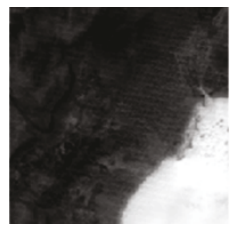

Playa

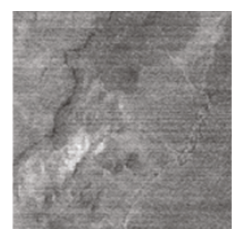

(a) LSOSP

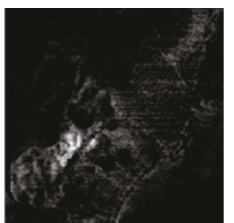

(b) NCLS

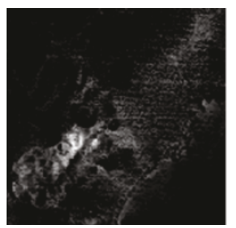

Rhyolite
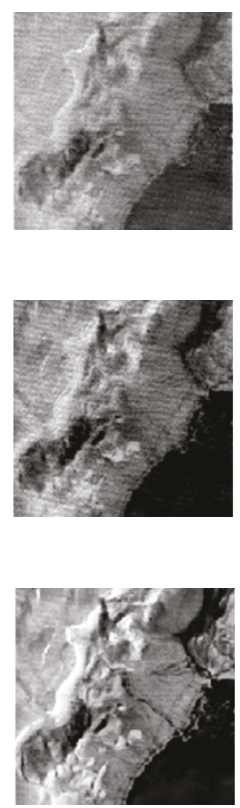

Shade
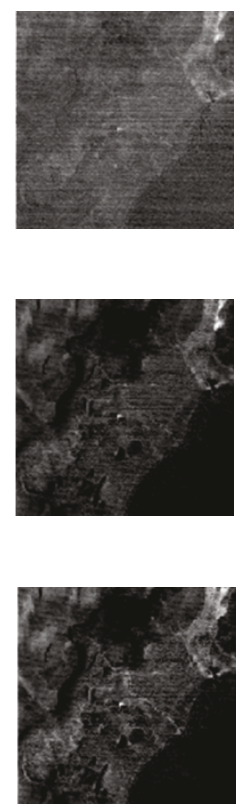

Vegetation

(c) FCLS

Figure 35: Unmixed results by unsupervised LSU, supervised LSOSP, NCLS, and FCLS using the five signatures in Figure 28(b).

\section{Conclusion}

Unsupervised spectral target analysis for hyperspectral data exploitation is very challenging since many unknown signal sources which cannot be visually inspected or obtained by prior knowledge can now be uncovered hyperspectral imaging sensors. This paper presents unsupervised spectral target analysis from a statistical signal processing view point in the sense of intrapixel spectral information across the acquired wavelength range. The knowledge used to perform unsupervised spectral target analysis is obtained directly from the data a posteriori without pre-assumed prior 
knowledge. In particular, the spectral targets of interest in this paper are specified by sample intrapixel spectral information statistics (SIS) which characterizes spectral targets into two categories, 2nd order spectral targets, referred to as background pixels and high-order spectral targets, referred to as target pixels. Additionally, in order to generate spectral targets in these two categories, an unsupervised spectral target finding algorithm is developed for this purpose where three least squares-based unsupervised linear spectral unmixing techniques, ATGP, UNCLS, and UFCLS are used for finding spectral targets of interest. Despite the fact that many algorithms have been designed and developed for supervised target analysis with the target knowledge assumed to be known or provided a priori, very little has been done in unsupervised target analysis. Unfortunately, in real applications supervised target analysis is generally not practical because the supervised target knowledge is either difficult to obtain or may not be reliable by prior knowledge. Under such circumstance unsupervised target analysis is more realistic and applicable to real world problems. In order to validate and substantiate the unsupervised spectral target analysis three applications are considered where two sets of experiments using custom-designed simulated synthetic images as well as real images are conducted for performance evaluation. The experimental results clearly show that unsupervised spectral target analysis generally performs significantly better than supervised target analysis in real applications. As a concluding remark, it should be noted that the proposed USTFA-based unsupervised target analysis is suitable for spectral targets that are characterized by sample intrapixel spectral information statistics. It is particularly useful and effective for very high spatial and spectral resolution hyperspectral images. Nevertheless, it is not a one-size-fit-all technique for hyperspectral imagery. For example, it may not be appropriate to use our proposed technique to analyze urban scenes which are complex and are heavily mixed by unknown clutters and interferers.

\section{Acknowledgment}

The work of C.-I Chang was supported by the National Science Council in Taiwan under NSC 98-2811-E-005-024 and NSC 98-2221-E-005-096.

\section{References}

[1] R. A. Schewengerdt, Remote Sensing: Models and Methods for Image Processing, Academic Press, Boston, Mass, USA, 2nd edition, 1997.

[2] C.-I Chang, Hyperspectral Imaging: Techniques for Spectral Detection and Classification, Kluwer Academic/Plenum Publishers, New York, NY, USA, 2003.

[3] C.-I Chang and M. Hsueh, "Characterization of anomaly detection in hyperspectral imagery," Sensor Review, vol. 26, no. 2, pp. 137-146, 2006.

[4] H. V. Poor, An Introduction to Signal Detection and Estimation, Springer, New York, NY, USA, 2nd edition, 1994.

[5] H. Ren and C.-I Chang, "Automatic spectral target recognition in hyperspectral imagery," IEEE Transactions on Aerospace and Electronic Systems, vol. 39, no. 4, pp. 1232-1249, 2003.
[6] C.-I Chang and D. Heinz, "Constrained subpixel target detection for remotely sensed imagery," IEEE Transactions on Geoscience and Remote Sensing, vol. 38, no. 3, pp. 1144-1159, 2000.

[7] D. C. Heinz and C.-I Chang, "Fully constrained least squares linear spectral mixture analysis method for material quantification in hyperspectral imagery," IEEE Transactions on Geoscience and Remote Sensing, vol. 39, no. 3, pp. 529-545, 2001.

[8] J. C. Harsanyi and C.-I Chang, "Hyperspectral image classification and dimensionality reduction: an orthogonal subspace projection approach," IEEE Transactions on Geoscience and Remote Sensing, vol. 32, no. 4, pp. 779-785, 1994.

[9] J. J. Settle, "On the relationship between spectral unmixing and subspace projection," IEEE Transactions on Geoscience and Remote Sensing, vol. 34, no. 4, pp. 1045-1046, 1996.

[10] C.-I Chang, "Further results on relationship between spectral unmixing and subspace projection," IEEE Transactions on Geoscience and Remote Sensing, vol. 36, no. 3, pp. 1030-1032, 1998.

[11] http://aviris.jpl.nasa.gov/.

[12] B. Thai and G. Healey, "Invariant subpixel material detection in hyperspectral imagery," IEEE Transactions on Geoscience and Remote Sensing, vol. 40, no. 3, pp. 599-608, 2002.

[13] J. W. Boardman, "Geometric mixture analysis of imaging spectrometery data," Proceedings of the International Geoscience and Remote Sensing Symposium, vol. 4, pp. 2369-2371, 1994.

[14] M. E. Winter, "N-finder: an algorithm for fast autonomous spectral endmember determination in hyperspectral data," in Image Spectrometry V, vol. 3753 of Proceedings of the SPIE, pp. 266-277, 1999.

[15] A. A. Green, M. Berman, P. Switzer, and M. D. Craig, "A transformation for ordering multispectral data in terms of image quality with implications for noise removal," IEEE Transactions on Geoscience and Remote Sensing, vol. 26, no. 1, pp. 65-74, 1988.

[16] C.-C. Wu, C. S. Lo, and C.-I Chang, "Improved process for use of a simplex growing algorithm for endmember extraction," IEEE Geoscience and Remote Sensing Letters, vol. 6, no. 3, pp. 523-527, 2009.

[17] C.-I Chang, "Target signature-constrained mixed pixel classification for hyperspectral imagery," IEEE Transactions on Geoscience and Remote Sensing, vol. 40, no. 5, pp. 1065-1081, 2002.

[18] I. S. Reed and X. Yu, "Adaptive multiple-band CFAR detection of an optical pattern with unknown spectral distribution," IEEE Transactions on Acoustics, Speech, and Signal Processing, vol. 38, no. 10, pp. 1760-1770, 1990.

[19] C.-I Chang, X.-L. Zhao, M. L. G. Althouse, and J. J. Pan, "Least squares subspace projection approach to mixed pixel classification for hyperspectral images," IEEE Transactions on Geoscience and Remote Sensing, vol. 36, no. 3, pp. 898-912, 1998.

[20] C.-I Chang, C. Wu, W. Liu, and Y. C. Ouyang, "A growing method for simplex-based endmember extraction algorithms," IEEE Transactions on Geoscience and Remote Sensing, vol. 44, no. 10, pp. 2804-2819, 2006. 